\title{
Information Flow and Price Discovery Dynamics by
}

Lei Wu Beihang University

Kuan Xu Dalhousie University

\author{
Qingbin Meng \\ Renmin University of China
}

Working Paper No. 2020-02

May o7, 2020

\section{DEPARTMENT OF ECONOMICS}

DALHOUSIE UNIVERSITY

6214 University

Avenue PO Box 15000

Halifax, Nova Scotia, CANADA

$\mathrm{B}_{3} \mathrm{H} 4 \mathrm{R} 2$ 


\title{
Information Flow and Price Discovery Dynamics
}

\author{
Lei Wu • Kuan Xu • Qingbin Meng
}

January 2020

\begin{abstract}
Non-homogeneous and time-varying information flow that affects the price discovery processes within and across markets is a common occurrence in reality but is often neglected in the literature of price discovery. To analyze such information flow within and across markets, we propose a new strategy with a new dynamic price discovery measure. We use this strategy to test the efficient home market hypothesis and the sector effect hypothesis based on the intraday data of the 115 stocks cross-listed and traded in the Canadian and U.S. stock markets. We find that the Canadian stock market is more efficient in price discovery for the Canadian stocks cross-listed in the U.S. stock market. A higher trading volume in the Canadian market makes price discovery in that market more efficient. The Canadian stock market is more efficient in price discovery for stocks in the basic materials sector but not in the technology and financial sectors. The NYSE Alternext is more efficient for junior stocks while the NASDAQ is more efficient for technology stocks.
\end{abstract}

JEL Codes: C5, F3, G1

Keywords: financial markets, price discovery, information flow

Theme Keywords: market microstructure, financial econometrics, financial mathematics

Lei $\mathrm{Wu}$

School of Economics and Management, Beihang University, No.37 Xueyuan Road, Beijing 100191, Email: l.wu@buaa.edu.cn

E-mail: l.wu@buaa.edu.cn

Kuan Xu (Corresponding Author)

Department of Economics, Dalhousie University, PO Box 15000, Halifax, NS, Canada B3H 4R2 \& Department of Economics,

Sobey School of Business, Saint Mary's University, 923 Robie Street, Halifax, NS, Canada B3H 3C3

E-mail: kuan.xu@dal.ca

Qingbin Meng

School of Business, Renmin University of China, No. 59 Haidian Road, Beijing, China 100872

E-mail: mengqingbin@rbs.ruc.edu.cn 


\section{Introduction}

Price discovery is one of the fundamental functions of financial markets. Cross listing of country-specific stocks in multiple stock markets across countries has recently become a notable trend. Through price discovery processes, all information will be eventually integrated into market prices but each market price may be exposed, and react with its own speed, to various shocks from within or across markets over time. Therefore, it is of great interest to understand price discovery processes within and across these markets over time.

The existing literature has examined price discovery for stocks in multiple markets both in one country (Hasbrouck 1995, 2003; Harris, et al. 2002) and in multiple countries (Werner and Kleidon 1996; Hupperets and Menkveld 2002; Eun and Sabherwal 2003; Grammig et al. 2005; Agarwal et al. 2007; Menkveld 2008; Otsubo 2014; Frijns et al. 2015a, 2015b, 2018). Among the latter, some authors (Werner and Kleidon 1996; Hupperets and Menkveld 2002; Grammig et al. 2005; Agarwal et al. 2007; Menkveld 2008; Otsubo 2014) focus on nonsynchronous markets across countries while some others (Eun and Sabherwal 2003; Frijns et al. 2015a, 2015b, 2018) examine synchronous markets across countries 1 Most studies focusing on synchronous markets are based on the standard structural model, or its variant, in which there are one permanent shock and one transitory shock in two markets. These studies use the vector error correction model (VECM) and contemporaneous correlations of some kind among shocks, such as the information share (in terms of variance decomposition), or the component share (in terms of factor weights), or both in absolute or relative terms, across two markets to infer the role of a market in price discovery.

One central inquiry is on the questions of how integrated these markets are and which market, home or foreign, plays a more important role in price discovery. The overwhelming evidence suggests that price discovery should take place mostly in the home market (referred to as the efficient home market hypothesis) but, as these markets become more integrated with more barriers removed, both international and home markets would have a shared role (Solnik 1996; Bacidore and Sofianos 2002; Kryzanowski and Zhang 2002; Eun and Sabherwal 2003; Frijns et al. 2015a, 2015b, 2018). Following Eun and Sabherwal (2003) ${ }^{2}$ based on more recent intraday data of the 115 stocks cross-listed in the Canadian and U.S. markets ${ }^{3}$ we find that the ratio of the error correction coefficients would change as the frequencies of the intraday data are varied and, therefore, this ratio does not provide a consistent evidence across different intraday data frequencies. At higher frequencies, the ratio indicates that the Canadian stock market is the dominant player in price discovery but, at lower frequencies, it suggests otherwise. Therefore, it is desirable to re-examine the methodology and any new evidence on the efficient home market hypothesis.

In addition, many scholars note that price discovery efficiency may vary across a range of factors, in particular across sectors, in the multi-market setting. For example, Kryzanowski and Zhang (2002) find the institutional differences affect the trade execution price/costs in the Canadian and U.S stock markets. Eun and Sabherwal (2003) show that price discovery efficiency could vary by sectors, trading volume, medium-sized trades, and market-cap. Lee et al. (2014) note that industries may have different comovements that will affect returns of cross-listed stocks. Frijns et al. (2015a, 2018) find that liquidity, which may vary across sectors as well, can be an important factor contributing to price discovery. The Canadian TSX is more specialized in the basic material sector while the U.S. NYSE Alternext and NASDAQ are more specialized in junior stocks and technology stocks, respectively. Therefore, it is desirable to examine any evidence on the sector effect hypothesis which states that price discovery for stocks in certain sectors is more efficient in the market that specializes in those sectors.

Apparently, if one permanent shock and one transitory shock arrive in two markets simultaneously, the ratio of error correction coefficients should not change over various intraday data frequencies as the two market prices are exposed to the same set of shocks over all frequencies. However, if market-specific permanent shocks, which jointly affect the fundamental value of a stock, differ in arriving time, distribution, and time taken to get absorbed within and across the two markets, the ratio of the error correction coefficients can vary over various intraday data frequencies. This finding from simulations 4 prompts us to look for alternative measures to the ratio of the error correction coefficients.

\footnotetext{
1 There is another literature on price discovery on dual class stocks (seeWang and Yang 2015). We do not study how to price dual class stocks that have different voting rights.

2 This approach is quite consistent with that of Gonzalo and Granger (1995).

3 Out of these 115 stocks, 106 are Canadian stocks and 9 are the U.S. stocks.

4 Due to the space limitation, we do not report the simulation design and results in this paper.
} 
A number of alternative measures to the ratio of the error correction coefficients are proposed and actively used in the literature. The information share (IS) measure based on the VECM framework reflects one market's share of the total price variance in two markets (see Hasbrouck 1995) $5^{5}$ The component share (CS) measure based on the VECM framework reflects the weight of one market price in the factor defined by Gonzalo and Granger (1995) relative to the total weights of two market prices (see Booth et al. 1999; Chu et al. 1999; Harris et al. 2002). The information leadership (ILS) measure combines IS with CS (see Yan and Zivot 2010). Putnins (2013) conducts a comprehensive assessment of IS, CS, and ILS and notes that all three measures are based on the standard structural model that contains one permanent shock and one transitory shock in two markets or two market-specific permanent shocks of the same distribution and two market-specific transitory shocks arriving simultaneously 6

To evaluate information flow and dynamic price discovery processes within and across markets, we first extend the standard structural model to accommodate the often-neglected non-homogeneous and time-varying innovations (different shocks differ in arriving time, distribution, and time taken to get absorbed within and across markets) ${ }^{7}$ Corresponding to this extended model, we then propose a strategy for identifying information channels within and across markets at different intraday data frequencies and design a dynamic price discovery measure that is sensitive to non-homogeneous and time-varying information flow within and across markets over time. To test the efficient home market and sector effect hypotheses, we finally analyze, in a panel data model, price discovery efficiency measures within and across markets at various intraday data frequencies for 115 stocks with reference to control variables such as country, market capitalization, medium-trades, volume, sector, and exchanges.

This paper offers a number of interesting findings. Using our dynamic price discovery measures within and across the Canadian and U.S. stock markets, we find that the Canadian stocks are exposed more to the information channels from the Canadian to the U.S. market than to those from the U.S. to the Canadian market. We find that innovations affect the price upon impact with large pricing discrepancies and that the market tends to over-react to the innovations coming from within but under-react to innovations from outside. We note that these pricing discrepancies vanish over time at various intraday data frequencies. Using the panel data model, we analyze the factors affecting price discovery efficiency within and across the markets at various intraday data frequencies. We note that price discovery for the Canadian stocks tends to be more efficient within that market. A higher trading volume in the Canadian home market makes price discovery in that market more efficient. Price discovery for stocks in the basic materials sector is more efficient in the Canadian market but stocks in the technology and financial sectors do not have any edge in price discovery efficiency. Price discovery for junior stocks in the NYSE Alternext and that for technology stocks in the NASDAQ are more efficient under certain conditions.

The remainder of this paper is organized as follows. In Section 2 we discuss the research methodology. Section 3, we present the data and empirical findings. In Section 4, we make concluding remarks.

\section{Research Methodology}

In this section, we focus on methodological issues and explain how to identify information channels, construct a dynamic price discovery measure, and use the panel data model to analyze price discovery efficiency within and across markets at different intraday data frequencies for our key research questions.

2.1 Price behaviors in the reduced-form and extended structural models

Let the Canadian and U.S. log prices of the stock be $p_{c n, t}$ and $p_{u s, t}$, respectively. Let the Canadian TSX and the U.S. S\&P 500 market indices in $\log$ be $p_{i d c n, t}$ and $p_{i d u s, t}$, respectively. We use $\mathbf{p}_{t}=\left[p_{c n, t}, p_{u s, t}, p_{i d c n, t}, p_{i d u s, t}\right]^{\prime}$ to denote the vector of $\log$ prices and indices.

\footnotetext{
5 As Yan and Zivot (2010) note, this measure may not be unique.

6 Frijns and Schotman (2009) propose a structural model in tick time for the quote setting dynamics on NASDAQ. Otsubo (2014) proposes a structural model and three price discovery measures based on the structural model but the model is only suitable to non-overlapping markets.

7 For example, Yeh et al. (2016) show that stock sales timing and profitability of insiders are highly correlated.
} 
According to the Wold representation theorem, the stock and market index returns in $\Delta \mathbf{p}_{t}=\mathbf{p}_{t}-\mathbf{p}_{t-1}$ have a multivariate moving average (MA) representation, which we call the reduced-form model:

$$
\Delta \mathbf{p}_{t}=\Psi(L) \mathbf{e}_{t}=\mathbf{e}_{t}+\boldsymbol{\Psi}_{1} \mathbf{e}_{t-1}+\boldsymbol{\Psi}_{2} \mathbf{e}_{t-2}+\cdots,
$$

where $\boldsymbol{\Psi}(L)=\sum_{k=0}^{\infty} \boldsymbol{\Psi}_{k} L^{k}$ with $\boldsymbol{\Psi}_{0}=\mathbf{I} . \boldsymbol{\Psi}(L)$ is a $4 \times 4$ polynomial matrix in lag operator $L$. The error terms in $\mathbf{e}_{t}$ are not necessarily independent among themselves. They represent various combinations of market-specific innovations and market-specific idiosyncratic noises. By the Granger representation theorem, the $4 \times 1$ price vector $\mathbf{p}_{t}$ is said to be cointegrated with rank $r$ if $\boldsymbol{\Psi}(1)$ is of rank $(n-r)$, and there exist two $n \times r$ matrices, $\alpha$ and $\beta$, both of rank $r$, such that $\beta^{\prime} \boldsymbol{\Psi}(1)=\mathbf{0}$ and $\boldsymbol{\Psi}(1) \alpha=\mathbf{0}$. The column of $\beta$ is the cointegrating vector and the column of $\alpha$ contains the error correction coefficients 8

Based on the law of one price for each stock and the fact that the two broad stock market indices represent different underlying assets, we expect that $r=1$. Here, $\beta$ is a $4 \times 1$ vector defined as $\beta=\left[\beta_{c n}, \beta_{u s}, \beta_{\text {idcn }}, \beta_{\text {idus }}\right]^{\prime}$, and $\alpha$ is a $4 \times 1$ vector defined as $\alpha=\left[\alpha_{c n}, \alpha_{u s}, \alpha_{i d c n}, \alpha_{i d u s}\right]^{\prime}$, where the subscripts $c n$ and us denote respectively the Canadian and U.S. stocks while the subscripts $i d c n$ and $i d u s$ denote respectively the Canadian and U.S. stock market indices. As we normalize $\beta_{c n}$ to 1 , we expect, and also have verified empirically later, $\beta=[1,-1,0,0]^{\prime}$ and that $\alpha_{i d c n}$ and $\alpha_{i d u s}$ in vector $\alpha$ are close to zero. This is because $p_{c n, t}$ and $p_{u s, t}$ must respond to each other to ensure the law of one price but the broad market indices $p_{i d c n, t}$ and $p_{i d u s, t}$ do not adjust to the individual stock prices $p_{c n, t}$ and $p_{u s, t}$ in the two markets. $\alpha_{c n}$ and $\alpha_{u s}$ are the error correction coefficients, the ratio of which in absolute value $\left(\frac{\left|\alpha_{c n}\right|}{\left|\alpha_{u s}\right|}\right)$ is used in analyzing price discovery by Eun and Subherwal (2003) and others.

The reduced-form model may demonstrate different properties depending on underlying structural models that generate the data. In the standard structural model, the Canadian and U.S. stock prices $p_{c n, t}$ and $p_{u s, t}$ are the functions of the shock $m_{t}$, a random walk process driven by innovation $\eta_{t}$, and the market-specific idiosyncratic noises $e_{c n, t}$ and $e_{u s, t}$, respectively:

$$
\begin{aligned}
p_{c n, t} & =m_{t}+e_{c n, t}, \\
p_{u s, t} & =m_{t}+e_{u s, t}, \\
m_{t} & =m_{t-1}+\eta_{t},
\end{aligned}
$$

where $\eta_{t} \sim\left(0, \sigma_{\eta}^{2}\right)$ and $e_{c n, t}, e_{u s, t} \sim\left(0, \sigma^{2}\right)$. To accommodate the often-neglected non-homogeneous and timevarying innovations, we extend the standard structural model to allow the two market prices of the same underlying stock to be exposed to market-specific shocks $\eta_{c n, t} \sim\left(0, \sigma_{\eta, c n}^{2}\right)$ and $\eta_{u s, \tau} \sim\left(0, \sigma_{\eta}^{2}, u s\right)$ that arrive at different points $(t$ and $\tau)$ in time, are of different dispersion sizes $\left(\sigma_{\eta, c n}^{2}\right.$ and $\left.\sigma_{\eta, u s}^{2}\right)$, and get absorbed with different lags $(m$ and $n)$ :

$$
\begin{aligned}
& p_{c n, t}=p_{c n, t-1}+\eta_{c n, t}+\eta_{u s, \tau-m}+e_{c n, t}-e_{c n, t-1}, \\
& p_{u s, t}=p_{u s, t-1}+\eta_{c n, t-n}+\eta_{u s, \tau}+e_{u s, t}-e_{u s, t-1} .
\end{aligned}
$$

Here, $\eta_{c n, t}$ is the innovation coming from the Canadian market at time $t$, while $\eta_{u s, \tau}$ is the innovation from the U.S. market at time $\tau$. $\tau$ may differ from $t$ and $\sigma_{\eta, c n}^{2}$ may differ from $\sigma_{\eta, u s}^{2}$. These two kinds of innovations may arrive at different points in time, are of different sizes, and get absorbed with different lags. Two marketspecific idiosyncratic noises $e_{c n, t}$ and $e_{u s, t}$ represent transitory shocks to the two market prices. The extended structural model is warranted as the news coverage, news readers' attention, and, therefore, information flow for the stocks cross-listed in the Canadian and U.S. stock markets may well be different across the markets 9 The standard structural model is the degenerated case of the extended structural model.

According to our extended structural model and Monte Carlo simulations ${ }^{10}$ if different market-specific innovations with different arrival times and different distributions drive the price discovery, the ratio $\frac{\left|\alpha_{c n}\right|}{\left|\alpha_{u s}\right|}$ will demonstrate varying patterns as the intraday data are sampled at different frequencies or the sampling of intraday data shifts forward in time from a particular point in time. More specifically, when, relative to the U.S. counterparts, the Canadian market innovations are of a smaller size, arrive more frequently, and may

\footnotetext{
8 The cointegration relations under consideration in this study have no deterministic components, which lie outside the cointegrating space in the sense of Johansen (1991).

9 See Chang (1998) for the role of insiders and Securities and Exchange Commission (2010) for the role of high frequency traders.

10 The simulation results are available upon request.
} 
get absorbed by the U.S. market price with a higher absorption speed, we observe that, at lower and lower frequencies, the ratio $\frac{\left|\alpha_{c n}\right|}{\left|\alpha_{u s}\right|}$ changes from a value less than 1 to a value greater than 1 . Further, when the sampling of intraday data shifts forward from a point in time at which the U.S. market innovations occur more densely than their Canadian counterparts do, we observe that the ratio $\frac{\left|\alpha_{c n}\right|}{\left|\alpha_{u s}\right|}$ changes from a value greater than 1 to a value less than 1. Therefore, we can use the observed evidence from the reduced-form model to infer the underlying characteristics of market-specific innovations. Clearly, the ratio $\frac{\left|\alpha_{c n}\right|}{\left|\alpha_{u s}\right|}$ should not change if the standard structural model captures accurately the true data generating process. The varying patterns of the ratio could result from different transmitting directions, occurring frequencies, and absorption speeds of the innovations within and across markets. To accommodate non-homogeneous and time-varying innovations implied by the extended structural model, we need to examine, over a range of intraday data frequencies, information channels and dynamic price discovery within and across the markets.

\subsection{Identification of information channels}

The error terms in $\mathbf{e}_{t}$ of the reduced-form model (1) are some combinations of innovations and idiosyncratic noises. To infer how innovations transmit within and across markets, we propose an identification strategy for finding information channels within and across markets at various intraday data frequencies.

Identified information channels show how different market-specific innovations affect the price discovery process within and across markets over different intraday data frequencies (e.g., from 1- to 65-minute frequencies). More specifically, $\mathbf{\Psi}(L)$ in the reduced-form model may vary subject to these varying intraday data frequencies. At a higher (lower) frequency, the parameters in the reduced-form model $\boldsymbol{\Psi}(L)$ would capture more (less) information transmissions leaving the error terms in $\mathbf{e}_{t}$ close to be (far away from) independent among themselves. Hence, by varying the intraday data frequency, we can infer the intensity of market-specific innovations based on the variations in both $\boldsymbol{\Psi}(L)$ and $\mathbf{e}_{t}$ of the reduced-form model and identify those information channels that are robust across these frequencies.

We use a SVAR model to identify the reduced-form model's error terms in $\mathbf{e}_{t}$ for the two stock returns and two market index returns as some combinations of i.i.d. structural shocks in $\mathbf{v}_{t}$, which correspond to independent market-specific innovations and market-specific idiosyncratic noises for both the stocks and market indices. Causal chains among structural shocks can be characterized as $\mathbf{A} \mathbf{e}_{t}=\mathbf{B v} t$, where $\mathbf{A}$ and $\mathbf{B}$ are both $4 \times 4$ matrices. The expression

$$
\mathbf{e}_{t}=\mathbf{A}^{-1} \mathbf{B v}_{t}
$$

provides the analytical links between the reduced-form model's error terms in $\mathbf{e}_{t}$ and the i.i.d. structural shocks in $\mathbf{v}_{t} . \mathbf{B}$ is a diagonal matrix with the diagonal elements being the standard deviations of structural shocks while $\mathbf{A}^{-1}$ represents information channels, by which i.i.d. structural shocks in $\mathbf{v}_{t}$ affect the error terms in $\mathbf{e}_{t}$.

To identify the structure of $\mathbf{A}$, we note that the stock market indices affect the constituting stock's prices in these markets contemporarily but not the other way around because the systematic risk in the stock market indices affects the returns on the constituting stock in these markets while the idiosyncratic risk in the constituting stock does not affect the returns on the stock market indices ${ }^{11}$ Therefore, as shown in equation (5), we impose zero restrictions on $\mathbf{A}$ at the cells $[3,1],[3,2],[4,1]$ and $[4,2]$ and the restrictions of 1 's on all diagonal elements of matrix $\mathbf{A}$ based on the argument of normalization.

$$
\mathbf{A}=\left[\begin{array}{cccc}
1 & A_{c n, u s} & A_{c n, i d c n} & A_{c n, i d u s} \\
A_{u s, c n} & 1 & A_{u s, i d c n} & A_{u s, i d u s} \\
0 & 0 & 1 & A_{\text {idcn }, \text { idus }} \\
0 & 0 & A_{\text {idus }, \text { idcn }} & 1
\end{array}\right]
$$

We use the significant estimates for $A_{u s, c n}$ and $A_{c n, u s}$ to infer the information channels "from the Canadian to the U.S. market" and "from the U.S. to Canadian market," respectively. Similarly, we use the estimates for $A_{i d u s, i d c n}$ and $A_{i d c n, i d u s}$ to infer the information channels between the two market indexes. The absolute value of the estimate shows the size of the information channel.

\footnotetext{
11 The asset pricing models suggest that the return of a particular stock in a market is priced in relation to the return of that market portfolio that contains this stock, among other factors, but not the other way around. Meantime, the law of one price also ensures the equilibrium between the two market prices of, therefore the resulting returns on, the same constituting stock. Hence, the returns on this stock in the two markets are jointly influenced by the two market indices.
} 


\subsection{A dynamic price discovery measure}

In this section, we use the permanent-transitory decomposition to construct a dynamic price discovery measure 12

We define $\mathbf{G}=\left[\begin{array}{c}\alpha_{\perp}^{\prime} \\ \beta^{\prime}\end{array}\right]$, where $\beta^{\prime}$ is a $1 \times 4$ matrix and $\alpha_{\perp}^{\prime}$ is a $3 \times 4$ matrix satisfying $\alpha_{\perp}^{\prime} \alpha=\mathbf{0}$, and substitute equation (4) into equation (1) for $\Delta \mathbf{p}_{t}$. Using $\mathbf{G}$, we decompose $\Delta \mathbf{p}_{t}$ into the long-run impact and pricing discrepancies:

$$
\begin{aligned}
\Delta \mathbf{p}_{t} & =\mathbf{\Psi}(L) \mathbf{G}^{-1} \mathbf{G A}^{-1} \mathbf{B} \mathbf{v}_{t} \\
& =\left[\mathbf{D}_{1}(L) \mathbf{D}_{2}(L)\right]\left[\begin{array}{c}
\alpha_{\perp}^{\prime} \\
\beta^{\prime}
\end{array}\right] \mathbf{A}^{-1} \mathbf{B} \mathbf{v}_{t} \\
& =\mathbf{D}_{1}(L) \alpha_{\perp}^{\prime} \mathbf{A}^{-1} \mathbf{B} \mathbf{v}_{t}+\mathbf{D}_{2}(L) \beta^{\prime} \mathbf{A}^{-1} \mathbf{B} \mathbf{v}_{t} \\
& =\underbrace{\mathbf{D}_{1}(1) \alpha_{\perp}^{\prime} \mathbf{A}^{-1} \mathbf{B} \mathbf{v}_{t}}_{\text {long-run impact denoted as }}+\underbrace{\left[\mathbf{D}_{1}(L) \alpha_{\perp}^{\prime} \mathbf{A}^{-1} \mathbf{B}-\mathbf{D}_{1}(1) \alpha_{\perp}^{\prime} \mathbf{A}^{-1} \mathbf{B}+\mathbf{D}_{2}(L) \beta^{\prime} \mathbf{A}^{-1} \mathbf{B}\right] \mathbf{v}_{t}}_{\text {pricing discrepancies denoted as } \mathbf{\Phi}^{*}(L) \mathbf{v}_{t} \text { with } \mathbf{\Phi}^{*}(1) \mathbf{v}_{t}=\mathbf{0}},
\end{aligned}
$$

The choice of $\mathbf{G}$ is motivated by the Granger representation theorem, which states that $\alpha_{\perp}^{\prime}$ and $\beta^{\prime}$ "knock out" the appropriate terms in the moving-average representations of $\Delta \mathbf{p}_{t}$ to isolate those components with the desired degree of persistence. The terms $\alpha_{\perp}^{\prime} \mathbf{A}^{-1} \mathbf{B} \mathbf{v}_{t}$ and $\beta^{\prime} \mathbf{A}^{-1} \mathbf{B} \mathbf{v}_{t}$ represent the shocks associated with permanent and transitory components of $\mathbf{p}_{t}$, respectively.

Note that the $4 \times 4$ matrix $\Psi(L) \mathbf{G}^{-1}$ is denoted as $\left[\mathbf{D}_{1}(L) \mathbf{D}_{2}(L)\right]$, of which $\mathbf{D}_{1}(L)$ contains the first 3 columns and $\mathbf{D}_{2}(L)$ contains the last 1 column. The polynomial matrices $\mathbf{D}_{1}(L)$ and $\mathbf{D}_{2}(L)$ are the responses of $\Delta \mathbf{p}_{t}$ to the shocks associated with permanent and transitory components, respectively. As the innovation associated with transitory components $\left(\beta^{\prime} \mathbf{A}^{-1} \mathbf{B} \mathbf{v}_{t}\right)$ has no long-run impact on $\mathbf{p}_{t}$, we expect $\mathbf{D}_{2}(1) \beta^{\prime} \mathbf{A}^{-1} \mathbf{B v}_{t}=$ 0. As the shocks associated with permanent components $\left(\alpha_{\perp}^{\prime} \mathbf{A}^{-1} \mathbf{B} \mathbf{v}_{t}\right)$ will have a long-run impact on $\mathbf{p}_{t}$, we expect $\mathbf{D}_{1}(1) \alpha_{\perp}^{\prime} \mathbf{A}^{-1} \mathbf{B} \mathbf{v}_{t} \neq \mathbf{0}$. Therefore, we decompose $\Delta \mathbf{p}_{t}$ into the long-run impact component $\left(\boldsymbol{\Phi} \mathbf{v}_{t}\right)$ and the pricing discrepancies component $\left(\Phi^{*}(L) \mathbf{v}_{t}\right)$. The initial impact of i.i.d structural shocks on $\Delta \mathbf{p}_{t}$ is $\mathbf{\Phi} \mathbf{v}_{t}+\boldsymbol{\Phi}^{*}(0) \mathbf{v}_{t}$, and the long-run impact of these shocks is $\mathbf{\Phi} \mathbf{v}_{t}$ as $\boldsymbol{\Phi}^{*}(1) \mathbf{v}_{t}=\mathbf{0}$. Thus, $\boldsymbol{\Phi}^{*}(0) \mathbf{v}_{t}$ captures initial pricing discrepancies that vanish over a long-enough period 13

Based on the above permanent-transitory decomposition, we propose our new dynamic price discovery measure when shocks are from market $j$ to market $i$ with time lag $t$, for $t=0,1,2, \ldots$,

$$
D_{i, j, t}=\frac{\boldsymbol{\Phi}_{i, j}+\boldsymbol{\Phi}^{*}(t)_{i, j}}{\boldsymbol{\Phi}_{i, j}}
$$

where $\boldsymbol{\Phi}_{i, j}\left(\boldsymbol{\Phi}^{*}(t)_{i, j}\right)$ is the $i, j$-th element of $\boldsymbol{\Phi}\left(\boldsymbol{\Phi}^{*}(t)\right)$. This measure is the ratio of the sum of the total impact on the price, including both the long-run impact and pricing discrepancies, in market $i$ caused by market $j$ to the long-run impact on price in market $i$ caused by market $j$ at time lag $t$. In Appendix, we provide a stylized example of $\boldsymbol{\Phi} \mathbf{v}_{t}$ and $\boldsymbol{\Phi}^{*}(L) \mathbf{v}_{t}$ for $L=1$ and the corresponding $D_{i, j, t}$.

The interpretation of the dynamic price discovery measure is as follows. When this ratio is 1 , there are no pricing discrepancies. When this ratio, $D_{i, j, t}$, differs from 1 , it indicates the existence of pricing discrepancies at time lag $t$. When this ratio is greater than 1 , there is an over-reaction of market $i$ to the shock from market $j$. When this ratio is greater than 0 but less than 1 , there is an under-reaction of market $i$ to the shock from market $j$. When this ratio takes a negative value, this indicates that market $i$ responds to the shock from market $j$ in the opposite direction and markets $i$ and $j$ do not share the same short-run common price movements. This ratio is also dynamic in nature. As time lag $t$ increases, when this ratio approaches 1 , this implies that pricing discrepancies vanish over time. The speed at which this ratio approaches 1 contains useful information on price discovery. When this ratio approaches 1 rapidly (slowly) as time lag $t$ increases, this implies a higher (lower) speed of information transmission from market $j$ to market $i$. In Appendix, we show the price discovery measures $D_{c n, u s, 0}$ and $D_{u s, c n, 0}$ given the specifications in the stylized example $(L=1)$ and how $\frac{\Phi_{c n, u s}}{\Phi_{u s, c n}}$ differs from $\frac{\left|\alpha_{c n}\right|}{\left|\alpha_{u s}\right|}$ when the market-specific shocks are the same $\left(v_{u s}=v_{c n}\right)$.

\footnotetext{
12 Please see Wu et al. (2015).

13 As proved by Wu et al. (2015), since $\alpha_{\perp}^{\prime} \mathbf{A}^{-1} \mathbf{B}$ still contains some transitory components, $\mathbf{D}_{1}(1) \alpha_{\perp}^{\prime} \mathbf{A}^{-1} \mathbf{B} \neq \mathbf{D}_{1}(0) \alpha_{\perp}^{\prime} \mathbf{A}^{-1} \mathbf{B}$ and $\left(\mathbf{D}_{1}(0) \alpha_{\perp}^{\prime} \mathbf{A}^{-1} \mathbf{B}-\mathbf{D}_{1}(1) \alpha_{\perp}^{\prime} \mathbf{A}^{-1} \mathbf{B}\right)$ is a part of the initial pricing discrepancies. For the detailed proof, see Wu et al. (2015).
} 
Different from the static price discovery measures in the literature, our dynamic price discovery measure $D_{i, j, t}$ is designed to capture the dynamic process through which innovations can fully transmit within and across the two markets. Behaving like an impulse response function, $D_{i, j, t}$ can measure initial price responsesovershooting, or undershooting, or counteracting - to market-specific shocks, pricing discrepancies' dynamics, and information transmission efficiency within and across the markets.

We can use this dynamic price discovery measure $D_{i, j, t}$ to identify market-specific shocks and their speeds of absorption within and across the markets at various intraday data frequencies. Corresponding to the extended structural model, our dynamic price discovery measure $D_{i, j, t}$ can be used in four cases among the Canadian and U.S. markets. That is, for $i, j=u s, c n, D_{i, j, t}$ is the dynamic price discovery measure for the impact of a shock from market $j$ on market $i$ at time lag $t$. Therefore, we can use $D_{c n, c n, t}, D_{u s, c n, t}, D_{c n, u s, t}$ and $D_{u s, u s, t}$ to measure the impacts of market-specific shocks within and across the markets. In addition, because $D_{u s, c n, t}$ and $D_{c n, u s, t}$ measure the cross-market information transmissions, any difference between these two measures as time lag $t$ increases will provide evidence on different absorption speeds for external shocks in the two markets.

We further construct the price discovery efficiency measure $S_{i, j}$ for the time path of $D_{i, j, t}$, which indicates the speed at which $D_{i, j, t}$ converges to 1 as time lag $t$ increases. To find the speed of convergence, we could use the standard deviation (std.dev) of $D_{i, j, t}$ 's over $t=1,2, \ldots, T \leq \infty$ as the smaller (greater) the standard deviation is, the more (less) quickly $D_{i, j, t}$ converges to one. Now denote the dynamic price discovery measure set $\mathbf{D}_{i, j}$ as the collection of $D_{i, j, t}$ 's over $t=1,2, \ldots, T \leq \infty$. Following Vives $(1993,1997)$, we define the price discovery efficiency measure $S_{i, j}$ as

$$
S_{i, j}=\frac{1}{\operatorname{std} \cdot \operatorname{dev}\left(\mathbf{D}_{i, j}\right)}
$$

which is inversely related to the standard deviation $\left(\operatorname{std} . \operatorname{dev}\left(\mathbf{D}_{i, j}\right)\right)$. The interpretation of $S_{i, j}$ is that the greater (smaller) the value that $S_{i, j}$ has, the more (less) efficiently innovations transmit from market $j$ to market $i$, and the more (less) quickly pricing discrepancies vanish in market $i$ in response to innovations in market $j 14$ The price discovery efficiency measure $S_{i, j}$ can be applied to the intraday data of various frequencies.

\subsection{Panel data model and hypothesis testing}

To evaluate price discovery efficiency and test the efficient home market and sector effect hypotheses for crosslisted stocks at various intraday data frequencies, we need to analyze $S_{i, j}$ 's in a panel data framework. In the model, we include the following factors as the control variables: (1) direction dummy variables for markets $i$ and $j,(2)$ the country where the company was incorporated and has its head office, (3) market-cap of the company, (4) medium trades, (5) trading volume, (6) the sector in which the company operates, and (7) the exchange where the stock is traded in the U.S. (the NYSE, NYSE Alternext, or NASDAQ) other than in the Canadian TSX. These control variables provide meaningful interpretations on how price discovery efficiency varies and are instrumental for testing the two hypotheses of interest.

The efficient home market hypothesis states that price discovery is more efficient for stocks in their home market. This is based on the bias in trading home stocks in the home market. This hypothesis was previously supported by Eun and Sabherwal (2003) but it's empirical evidence appears to be inconsistent as the intraday data of lower frequencies are examined. The sector effect hypothesis states that price discovery for stocks in certain sectors is more efficient in the market that specializes in those sectors. This hypothesis was previously supported by Eun and Sabherwal (2003) and Lee et al. (2014) but we do not know if a robust evidence for price discovery efficiency exits in some sectors across intraday data frequencies. It is hypothesized that price discovery efficiency for stocks in the basic materials sector should be more efficient in the Canadian TSX as it is specialized in the basic materials sector. Price discovery efficiency for junior stocks should be more efficient in the U.S. NYSE Alternext as it is specialized in junior stocks. Price discovery efficiency for stocks in the technology sector should be more efficient in the U.S. NASDAQ as it is specialized in the technology sector.

\section{Empirical Analysis}

In this section, we first discuss the data used for this research in subsection 3.1 and the behaviors of the two markets of various intraday data frequencies and sampling methods in subsection 3.2 . Then, in subsection 3.3 .

\footnotetext{
14 In the empirical research, we can specify a cutoff point $T$ at the time lag when pricing discrepancies vanish.
} 
we present our findings from the information channel analysis, and, in subsection 3.4 , we report the estimates of dynamic price discovery measure $D$ and price discovery efficiency measure $S$. Finally, in subsection 3.5 , we discuss the panel data model and the key findings.

\subsection{Data}

The stock markets in Canada and the U.S. are synchronous and highly integrated. Both the Canadian and U.S. stock markets are open to foreign investors and foreign corporations. Each country is the largest goods trading partner of the other. The two countries share the longest international border between them in the world and also share the same time zones. The news media in both Canada and the U.S. uses English ${ }^{15}$ The stock exchanges in both countries use the decimal pricing system ${ }^{16}$ The Canadian and U.S. stock exchanges have the identical open time (9:30 EST or 14:30 UTC) and close time (16:00 EST or 21:00 UTC). Similarities between the Canadian and U.S. stock markets provide a fertile environment for studying price discovery in synchronous markets.

We obtain our data from several sources. From the TMX Group's Internet portal, we retrieve the names of the Canadian stocks and non-Canadian stocks cross-listed and traded in both the Canadian stock exchange (TSX) and the U.S. stock exchanges (the NYSE, NYSE Alternext, and NASDAQ). All together we obtained 140 names of the stocks for which we have both intraday and daily data from February 14, 2016 to July 28, 2017.

We obtain further the corporation information from Finviz. The information includes the country in which the company is incorporated and has its head office, market-capitalization (market-cap) of the company, the sector in which the company operates, and the exchange in which a stock is listed and traded in the U.S. (the NYSE, NYSE Alternext, and NASDAQ). Out of these 140 companies, 126 are Canadian and 14 are American. These companies belong to various sectors such as basic materials, consumer goods, financial, health care, industrial goods, services, technology, and utility.

We obtain the intraday data for the period of February 14, 2016-July 28, 2017 for the 140 companies, the S\&P 500 and TSX market indices, and the U.S.-Canadian exchange rate from Google Finance backed by the Interactive Data Real-Time Services, Inc ${ }^{17}$ The intraday data contain time stamp, open, high, low, and close prices, and trading volume in both the Canadian and U.S. stock markets at the 1-minute frequency. The intraday data of lower frequencies can be sub-sampled from the 1-minute intraday data ${ }^{18}$ We have obtained the end-of-day data for the period of February 14, 2016-July 28, 2017 for the 140 companies from Yahoo Finance 19 The end-of-day data contain time stamp, open, high, low, close prices, and trading volume in Canadian and the U.S. stock markets at the end of trading day.

We note that some stocks are thinly and irregularly traded. We drop 25 companies (20 Canadian and 5 American stocks) whose trading occurs less than 20\% (78 minutes) of a trading day (390 minutes) in at least one of the two stock markets during the period of February 14, 2016-July 28, 2017 ${ }^{20}$ Therefore, we study the remaining 115 actively traded companies with 106 being Canadian and 9 being American. We note that the majority of the actively traded companies are Canadian companies.

As noted by Securities and Exchange Commission (2010) one of the most significant market structure developments in the recent years is the participants in automated exchanges from high frequency traders

\footnotetext{
15 Quebec's official language is French while English and French are official languages in Canada.

16 The Canadian exchanges adopted decimal pricing on April 15, 1996. The NASDAQ completed decimal pricing on March 12, 2001. The NYSE completed decimal pricing on September 11, 2001.

17 The data information can be found at https://www.google.com/googlefinance/disclaimer/ R API programmed by the authors is used to extract the intraday data every two weeks from February 14, 2016 to July 28, 2017. Google terminated Google Finance in mid-November 2017. We use the TSX 60 ETF and S\&P 500 ETF as the proxies for the market indices. For each actively traded stock, its 1-minute intraday data cover 391 data points (for 390 minutes) in each trading day, 385 trading days (for 15 months) in the entire sample, and in each of the two stock markets. That is, for each actively traded stock, there are about $301,070(=2 \times 391 \times 385)$ data points. Some stocks that are traded less frequently would have less data points over time across the two markets.

18 We select these frequencies (1-, 5-,10-,15-, 30-, and 65-minute frequencies) as they will not omit the critical end of day trading in the whole day which has 390 trading minutes. These frequencies can be multiplied by an integer to match 390 .

19 The $\mathrm{R}$ package BatchGetSymbol is used to extract the end-of-day data.

20 According to Heinkel and Kraus (1988), if a stock trades less than $20 \%$ of all trading days, it is considered a thinly traded stock. Using this benchmark in the intraday setting, if a stock trades less than $20 \%$ (78 minutes) of a trading day (390 minutes) in at least one of the two stock markets, it is considered a thinly traded stock.
} 
(HFTs), who trade and send in quotes in milliseconds." Brogaard et al. (2014) use millisecond trade and quote data to show that overall HFTs improve price efficiency by trading in the direction of permanent price changes and in the opposite direction of transitory pricing errors. HFTs can predict price changes over horizons of less than 3 to 4 seconds and their actions correct pricing errors at higher frequencies. In view of this significant development and the role of HFTs have played, if we focus on the price discovery efficiency based on permanent price changes and departures from such changes, we can still infer robust inference on price discovery efficiency based on the 1-minute trade data 21

Table 1 provides a snapshot of these 115 companies, among which 106 are Canadian and 9 are American. Sixty-six companies have their stocks traded in the NYSE, 30 companies have their stocks traded in the NYSE Alternext, and 19 companies' stocks are traded in the NASDAQ. The top three sectors by company counts are basic materials, technology, and financial sectors. The top three sectors by average company's market-cap are financial, services, and technology sectors. Out of the 115 companies, 70 companies are in the basic materials sector with the average capitalization of about U.S. $\$ 7.0$ billion. Twenty-eight of these 70 companies in the basic materials sector have their stocks traded in the NYSE Alternext and many of them are mid-cap and small-cap companies. However, out of the 115 companies, there are only 9 large-cap companies in the financial sector with the average capitalization of U.S. $\$ 54.20$ billion, among which, 8 companies have their stocks traded in the NYSE, 1 company has its stock traded in the NYSE Alternext, and no one has its stock traded in the NASDAQ.

(Please insert Table 1 about here)

3.2 Analysis of two markets across different frequencies and with different sampling methods

\subsubsection{Various frequencies}

In Table 2, we report the average estimates of cointegration vectors and error correction coefficient vectors for the 115 stocks at various intraday data frequencies. For each coefficient, we report the average estimate of the 115 stocks and the $5 \%$ and $95 \%$ percentiles of the statistically significant coefficient estimates in the parentheses. As expected, the average estimate of $\beta_{u s}$ is close to -1 at all frequencies, and the average estimates of $\beta_{i d c n}$ and $\beta_{i d u s}$ are close to zero at all frequencies. These findings confirm the law of one price for all individual stocks. The average estimates of $\alpha_{i d c n}$ and $\alpha_{i d u s}$ are also close to zero at all frequencies as expected. These findings show the insensitivity of the market indices to the law of one price for all individual stocks, which justifies our restrictions for the reduced-form model in equation (1).

\section{(Please insert Table 2 about here)}

As noted previously, because innovations are non-homogeneous and time-varying, there is a need for exploring intraday data at different frequencies to reveal more information about market-specific innovations, their arrival times, and their absorption speeds in relevant markets. We therefore analyze the intraday data at the 1-, 5-, 10-, 15-, 30-, and 65-minute frequencies and at the 1-day close and open frequencies. We find that the absolute values of the average $\alpha_{c n}$ and $\alpha_{u s}$ estimates increase as the frequency gets lower. The relative size of the two average error correction coefficients measured by $\frac{\left|\alpha_{c n}\right|}{\left|\alpha_{u s}\right|}$ increases from a value less than 1 at the 1-minute frequency to a value greater than 1 at the 15-minute frequency, reaching the highest value of 8.919 at the 1-day open frequency. These observations suggest that the price in the Canadian market tends to adjust more than its counterpart in the U.S. market at lower frequencies and that the price in the U.S. market tends to adjust more than its counterpart in the Canadian market at higher frequencies.

Our simulation results indicate that the ratio $\frac{\left|\alpha_{c n}\right|}{\left|\alpha_{u s}\right|}$ changes from a value less than 1 to a value great than 1 if the innovations of the Canadian market are of a smaller size, arrive at a higher frequency, and get absorbed by the U.S. market at a higher absorption speed ${ }^{22}$ The results also imply that, the U.S. market may gather and

21 Chiang and Fong (2001), Brooks et al. (2003), Adams et al. (2004), and Hautsch et al. (2011) examine how equity market prices and returns react, respectively, to the futures and option markets, unanticipated events, inflation news, and macroeconomic news using minute-by-minute data. Ellul (2006) analyzes the price discovery process across markets around large trades using five-minute frequency data. Chaboud et al. (2014) study how algorithmic trading affect the pricing efficiency of foreign exchange using minute-by-minute data. Hatheway et al. (2017) and Menkveld et al. (2017) study the impact of dark venues on price discovery using 1-minute data sampled from much higher frequency data.

22 The simulation results are available upon request. 
interpret more information at the open and end of the trading day, leading to a price adjustment mechanism at the open and end of the trading day somewhat different from that in the middle of the trading day.

\subsubsection{Various sampling methods}

For each of the selected samples of 60-, 30- 10-, and 5-minute frequencies, we shift the sampling of a particular frequency from a starting point forward, one minute at a time, from 1 to 31 shifts within the trading day ${ }^{23}$ The samples resulting from these sampling shifts have the following features: (1) as the starting point in time for the sampling shifts forward in a trading day, each sample has less exposure to the first (few) minute(s) of the trading day but more exposure to the last (few) minute(s) of the trading day and (2) due to different frequencies of these samples, the samples of the 60- and 30-minute frequency contain proportionally more data in the first (few) open minutes and last (few) close minutes while the samples of the 10- and 5-minute frequencies contain proportionally fewer data in first (few) open minutes and last (few) close minutes.

With these features in mind, we now examine Figures 1, 2, 3, and 4. Figures 1 and 2 show that for the intraday data of the 60- and 30-minute frequencies, $\frac{\left|\alpha_{c n}\right|}{\left|\alpha_{u s}\right|}>1$ at all 31 shifts. In particular, both Figures 1 and 2 show a flattened U-shape pattern. However, Figures 3 and 4 show that for the intraday data of the 10- and 5 -minute frequencies, $\frac{\left|\alpha_{c n}\right|}{\left|\alpha_{u s}\right|}<1$ at all 31 shifts. Because the intraday data of the 60- and 30-minute frequencies have more exposure to the first (few) open minute(s) and last (few) close minute(s) of the trading day than their 10- and 5-minute counterparts, the findings from Figures 1 and 2 confirm that the U.S. price contains more information at the first (few) open minute(s) and last (few) close minute(s) of the trading day and that the Canadian price makes more adjustments, at the first (few) open minute(s) and last (few) close minutes of the trading day, to match the U.S. price which carries more innovations at either the open or the end of the trading day.

(Please insert Figures 1, 2, 3, and 4 about here)

\subsection{Information channels}

In this subsection, we report the identified information channels within and across the Canadian and U.S. markets at different intraday data frequencies. At each intraday data frequency, the parameters at the positions [1,2] and [2,1] of $\mathbf{A}$ capture the information channels for the two stock prices "from the U.S. to the Canadian market" and "from the Canadian to the U.S. market," respectively. The parameters at the positions [3,4] and $[4,3]$ of A capture the information channels for the two market indices. These key parameters at different intraday data frequencies are useful for inferring information channels at these frequencies.

In the unrestricted model, the parameter matrix $\mathbf{A}_{u}$ simply takes the following pattern of information channels:

$$
\mathbf{A}_{u}=\left[\begin{array}{cccc}
1 & N a & N a & N a \\
0 & 1 & N a & N a \\
0 & 0 & 1 & N a \\
0 & 0 & 0 & 1
\end{array}\right]
$$

where 0 and 1 are restrictions 0 and 1 , respectively, and " $\mathrm{Na"} \mathrm{is} \mathrm{a} \mathrm{free} \mathrm{parameter.} \mathrm{In} \mathrm{the} \mathrm{restricted} \mathrm{models,}$ additional restrictions on $\mathbf{A}$ are imposed. On the positions [1,3], [1,4], [2,3] and [2,4] of matrix $\mathbf{A}$, there are four different positions on which only one 0 restriction could be imposed. On the positions $[1,2]$ and $[2,1]$ of matrix $\mathbf{A}$, there are two different positions on which only one 0 restriction could be imposed. On the positions $[3,4]$ and $[4,3]$ of matrix $\mathbf{A}$, there are two different positions on which only one 0 restriction could be imposed. Therefore, there could be total 16 patterns of restrictions imposed on matrix $\mathbf{A}$, denoted $\mathbf{A}_{i}, i=1, \ldots, 16$, as in Table 3

(Please insert Table 3 about here)

23 For example, when we use the intraday data of the 60-minute frequency, the data are sampled every 60 minutes starting from 9:30 till 360 minutes. We shift this sample from 9:30 EST to 9:31 EST, 9:32 EST, ..., 10:00 EST, respectively, and the last point from 15:30 EST to 15:31 EST, 15:32 EST, .., 16:00 EST for 31 shifts, respectively. 
Following the research strategy proposed in subsection 2.2 we identify those information channels supported by the data. For each stock, we estimate the unrestricted model with $\mathbf{A}_{u}$ and the restricted models with $\mathbf{A}_{i}$, $i=1, \ldots, 16$. We select the best fitted restricted model with $\mathbf{A}_{i}$ that has the highest $\log$-likelihood function value and then implement the likelihood ratio test for the validity of those additional restrictions imposed on $\mathbf{A}_{i}$ relative to $\mathbf{A}_{u}$. We then record the estimated $\mathbf{A}_{i}$ of the best fitted restricted model which could not be rejected by the likelihood ratio test. We implement the likelihood ratio tests for all 115 stocks across various intraday data frequencies. Each stock's data reveal its information channels embedded in $\mathbf{A}_{i}$ across various intraday data frequencies.

We summarize the identified information channels across the 115 stocks over 1-, 5-, 10-, 15-, 30-, and 65minute frequencies in Table 4. The table shows, based on the likelihood ratio tests, the numbers of stocks that have a specific pattern of information channels embedded in $\mathbf{A}_{i}$ at a particular frequency (one of 1-, 5-, 10-, 15-, 30-, and 65-minute frequencies). As shown in Table 4. the information channels are shared by some stocks but vary across stocks and different intraday data frequencies. Our testing results indicate that no stock based on the intraday data of various frequencies has the patterns of information channels embedded in $\mathbf{A}_{2}, \mathbf{A}_{4}, \mathbf{A}_{6}$, $\mathbf{A}_{8}, \mathbf{A}_{10}, \mathbf{A}_{12}, \mathbf{A}_{14}$, and $\mathbf{A}_{16}$. These estimated $\mathbf{A}_{i}$ 's share a common information channel-from the Canadian market index to the U.S. market index. This implies that the U.S. market index has significant influence on the Canadian market index but not the other way around.

\section{(Please insert Table 4 about here)}

In Table 4, we classify the information channels into either "from Canada to the U.S." or "from the U.S. to Canada.". For example, the stocks with information channels "from Canada to the U.S." are the stocks that support $\mathbf{A}_{3}, \mathbf{A}_{7}, \mathbf{A}_{11}$, and $\mathbf{A}_{15}$ containing non-zero $A_{u s, c n}$ in $\mathbf{A}_{i}$. By the same logic, the stocks with information channels "from the Canada to the U.S." are the stocks that support $\mathbf{A}_{1}, \mathbf{A}_{5}, \mathbf{A}_{9}$, and $\mathbf{A}_{13}$ containing nonzero $A_{c n, u s}$ in $\mathbf{A}_{i}$. Among all these selected patterns of information channels, $\mathbf{A}_{11}$ demonstrates the highest information channels "from Canada to the U.S." across various intraday data frequencies. Overall, Table 4 shows that the information channels "from Canada to the U.S." are significantly more than those "from the U.S. to Canada." The interpretation of this observation is that while both the Canadian and U.S. markets play a role in price discovery, the Canadian market releases more information than the U.S. market does.

\subsection{Price discovery}

\subsubsection{Empirical evidence for dynamic price discovery}

Tables 56 report the average price discovery measures $D_{c n, c n, t}, D_{u s, c n, t}, D_{c n, u s, t}$, and $D_{u s, u s, t}$ and their corresponding standard errors for the 115 stocks at various time lags, based on the intraday data of 1-, 5-, 10-, $15-, 30-$, and 65-minute frequencies, respectively ${ }^{24}$

(Please insert Tables 5 6 about here)

Tables 5 . show that $D_{c n, c n, t}$ and $D_{u s, u s, t}$ tend to be greater than 1 when lag $t=0$ whereas $D_{c n, u s, t}$ and $D_{u s, c n, t}$ tend to be less than 1 when lag $t=0$. These results indicate that the market tends to over-react (under-react) to the innovations coming from within (from outside).

In Tables 5 6, the numbers in bold font are those average price discovery measures that first reach the range of $1 \pm 0.05$ as the number of time lags $(t)$ increases. This implies that, with enough time lags $(t=T)$, pricing discrepancies would vanish. Tables 56 show that, at various intraday data frequencies, information could be absorbed within and across the markets within 200 minutes or less on average. But the absorption time can be much shorter (20 minutes) as shown for Barrick Gold in the next subsection. $D_{u s, u s, t}$ and $D_{u s, c n, t}$ converge to 1 in about 160 minutes while $D_{c n, c n, t}$ and $D_{c n, u s, t}$ converge to 1 in about 190 minutes. These aggregate results indicate that the U.S. market absorbs information faster, on average, than the Canadian market does, although only marginally 25

\footnotetext{
24 We multiply time lag $t$ by the intraday data frequency in minutes to convert a particular number of time lags $t$ into the number of lags in minutes for the intraday data. For example, Table 5 provides the average and standard error of $D_{i, j, t}$ at intraday data of 5 -minute frequency, so the column $t=10$ in Table 5 corresponds to information transmission at lags of 50 minutes $(=10 \times 5$ minutes $)$.

25 The country difference observed here is based on the pairwise comparison across the two countries. The net effect of country differences may differ when all other factors are held constant as is the case in the panel data model discussed later.
} 
3.4.2 A case study of $D_{u s, c n, t}$ : Barrick Gold (ABX)

To supplement these aggregate results in Tables 5, 6, we show the dynamic price discovery measure for the U.S. price of Barrick Gold (Ticker Symbol: ABX for both the NYSE and TSX) 26 , a well-known Canadian company and the world largest gold mining company, in response to innovations from the Canadian market.

As shown in Figures 5 7, the price discovery measure of innovations transmitted from the Canadian to the U.S. market, $D_{u s, c n, t}$, converges to 1 over time and eventually reaches 1 at about 20 minutes based on the intraday data of the 10-, 5-, and 1-minute frequencies. This convergence is very robust across different intraday data frequencies. Therefore, there is a strong evidence for the price discovery efficiency in the U.S. market for Barrick Gold in response to innovations from the Canadian market.

(Please insert Figures 5,7 about here)

\subsubsection{Empirical evidence for price discovery efficiency}

Recall that $D_{i, j, t}$ over time is a function measuring dynamic price discovery to its fullness with innovations transmitted from market $j$ to market $i$ over time. We can summarize the function of $D_{i, j, t}$ over time to measure price discovery efficiency in terms of $S_{i, j}$. The greater (smaller) the value for $S_{i, j}$ is, the more (less) efficiently innovations transmit from market $j$ to market $i$, and the more (less) quickly pricing discrepancies vanish. Tables 78 provide the summary for $S_{i, j}$ 's, $i, j=c n, u s$, and their pairwise correlations at the 1-, 5-, 10-, 15-, 30-, and 65-minute frequencies. In Table 7, we find that across 1-65 minute frequencies, the values of $S_{c n, c n}$ (in bold font) are the highest in each row. This implies that the Canadian market is more efficient in absorbing innovations from within. We find that the values of $S_{u s, c n}$ (in bold font) are the second highest in each row. This implies that the U.S. market is also efficient in absorbing innovations from the Canadian market. This is consistent with our finding that the vast majority of the companies are registered in Canada where the information of these companies tends to be released more frequently.

\section{(Please insert Tables 7,8 about here)}

Table 8 reports the pairwise correlations among price discovery efficiency measures. The correlation coefficients between $S_{c n, c n}$ and $S_{u s, c n}$ and that between $S_{u s, u s}$ and $S_{c n, u s}$ are strong and positive (in bold font) at 1-, 5-, 10-, 15-, 30-, and 65-minute frequencies. These indicate that if price discovery is more efficient within a market, it will also be more efficient when innovations transmitting from that market to the other market. This is true for both the Canadian and U.S. markets.

\subsection{Factors affecting price discovery efficiency}

In our panel data model, the key variables of interest are price discovery efficiency measures $\log \left(S_{i, j}\right)$ 's within and across markets $i$ and $j(i, j=c n, u s)$ for the 115 stocks at 1-, 5-, 10-, 15-, 30-, and 65-minute frequencies ${ }^{27}$ The panel data model also includes a set of independent variables, which are explained in detail below.

The panel data model includes three direction dummy variables as factors. With the price discovery efficiency measure within Canada $\left[\log \left(S_{c n, c n}\right)\right]$ being the baseline case, the first direction dummy variable is defined as $D_{S_{u s, c n}}=1$ if $\log \left(S_{i, j}\right)$ is $\log \left(S_{u s, c n}\right)$ and $D_{S_{u s, c n}}=0$ otherwise. The second direction dummy variable is defined as $D_{S_{c n, u s}}=1$ if $\log \left(S_{i, j}\right)$ is $\log \left(S_{c n, u s}\right)$ and $D_{S_{c n, u s}}=0$ otherwise. The third direction dummy variable is defined as $D_{S_{u s, u s}}=1$ if $\log \left(S_{i, j}\right)$ is $\log \left(S_{u s, u s}\right)$ and $D_{S_{u s, u s}}=0$ otherwise.

The panel data model includes one country dummy variable. With the U.S. being the baseline case, the country dummy is defined as Canada $=1$ for Canada and Canada $=0$ otherwise ${ }^{28}$

The panel data model includes two exchange dummy variables. While all the 115 stocks are traded in the TSX in Canada, they are also traded in the different U.S. stock exchanges. With the NYSE being the baseline case, the first exchange dummy variable is defined as Alternext $=1$ if the exchange is the NYSE Alternext and

\footnotetext{
26 The ticker symbol for Barrick Gold became GOLD on January 2, 2019 after Barrick and Randgold merged into one corporation still named as Barrick Gold.

27 We use $\log (S)$ as this logarithmic transformation gives the panel model a better fit.

28 Among the 115 companies studied, 106 are Canadian and 9 are American. Therefore, our inference would focus on Canadian corporations and their stocks.
} 
Alternext $=0$ otherwise. The second exchange dummy variable is defined as NASDAQ $=1$ if the exchange is NASDAQ and NASDAQ $=0$ otherwise.

The panel data model includes three sector dummy variables ${ }^{29}$ The first sector dummy variable is defined as Basic $=1$ if the sector is basic materials and Basic $=0$ otherwise. The second sector dummy variable is defined as Technology $=1$ if the sector is technology and Technology $=0$ otherwise. The third sector dummy variable is defined as Financial $=1$ if the sector is financial and Financial $=0$ otherwise. All other sectors (consumer goods, health care, industrial goods, services, and utilities) are included in the baseline case.

In addition, the panel data model includes the following continuous variables in logarithmic forms: market-

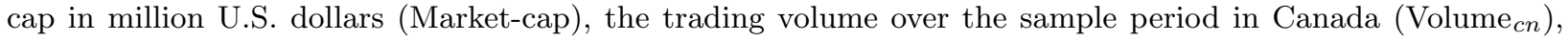
and the trading volume over the sample period in the U.S. (Volume $u s$ ). The panel data model also includes the

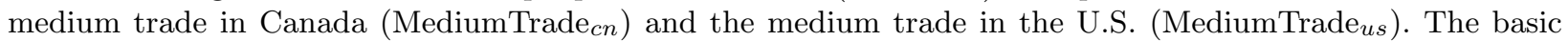
statistics of these continuous variables are given in Table 9. Market-cap for a cross-listed stock is measured in million U.S. dollars and is identical across the Canadian and U.S. markets. We note that the total numbers of shares traded in the sample period are much higher on average, and swing in a much wider range, in the U.S. market than in the Canadian market. Similarly, the numbers of shares traded per minute are much higher on average, and swing in a much wider range, in the U.S. market than in the Canadian market. MediumTrade is the standard deviation of the minute-by-minute trading volumes and is a measure for the proportion of medium trades ${ }^{30}$ The smaller the standard deviation, the more medium trades dominate extreme-very large or vary small - trades. The average and standard deviation of MediumTrade is lower in the U.S. market than in the Canadian market. That implies that there are more medium trades in the U.S. market than those in the Canadian market.

(Please insert Table 9 about here)

To accommodate information transmission in various directions, the panel data model also includes every cross product of each direction dummy variable (such as $D_{S_{c n, u s}}, D_{S_{u s, c n}}$, and $D_{S_{u s, u s}}$ ) and each control variable (such as Market-cap, Canada, MediumTrade $c n$, MediumTrade $_{u s}$, Volume $_{c n}$, Volume $u s$, Basic, Technology, Financial, Alternext, and NASDAQ).

The panel data model for the 115 stocks across 1-, 5-, 10-, 15-, 30-, and 65-minute frequencies accommodates inherent clusters within each stock and at each frequency. With these two clusters taken care of, the panel data model for $\log \left(S_{i, j}\right), i, j=c n, u s$, can be specified as follows:

$$
\begin{aligned}
& \log \left(S_{i, j}\right)=\beta_{0}+\beta_{c n, 1} \text { Canada }+\beta_{c n, 2} D_{S_{c n, u s}} * \text { Canada }+\beta_{c n, 3} D_{S_{u s, c n}} * \text { Canada } \\
& +\beta_{c n, 4} D_{S_{u s, u s}} * \text { Canada }+\beta_{m c, 1} \text { Market-cap }+\beta_{m c, 2} D_{S_{c n, u s}} * \text { Market-cap } \\
& +\beta_{m c, 3} D_{S_{u s, c n}} * \text { Market-cap }+\beta_{m c, 4} D_{S_{u s, u s}} * \text { Market-cap }+\beta_{m t c n, 1} \text { MediumTrade }_{c n} \\
& +\beta_{m t c n, 2} D_{S_{c n, u s}} * \operatorname{MediumTrade}_{c n}+\beta_{m t c n, 3} D_{S_{u s, c n}} * \operatorname{MediumTrade}_{c n}+\beta_{m t c n, 4} D_{S_{u s, u s}} * \operatorname{MediumTrade}_{c n} \\
& +\beta_{m t u s, 1} \text { MediumTrade }_{u s}+\beta_{m t u s, 2} D_{S_{c n, u s}} * \text { MediumTrade }_{u s}+\beta_{m t u s, 3} D_{S_{u s, c n}} * \text { MediumTrade }_{u s} \\
& +\beta_{m t u s, 4} D_{S_{u s, u s}} * \text { MediumTrade }_{u s}+\beta_{v c n, 1} \text { Volume }_{c n}+\beta_{v c n, 2} D_{S_{c n, u s}} * \text { Volume }_{c n} \\
& +\beta_{v c n, 3} D_{S_{u s, c n}} * \text { Volume }_{c n}+\beta_{v c n, 4} D_{S_{u s, u s}} * \text { Volume }_{c n}+\beta_{v u s, 1} \text { Volume }_{u s} \\
& +\beta_{v u s, 2} D_{S_{c n, u s}} * \text { Volume }_{u s}+\beta_{v u s, 3} D_{S_{u s, c n}} * \text { Volume }_{u s}+\beta_{v u s, 4} D_{S_{u s, u s}} * \text { Volume }_{u s} \\
& +\beta_{b s, 1} \text { Basic }+\beta_{b s, 2} D_{S_{c n, u s}} * \text { Basic }+\beta_{b s, 3} D_{S_{u s, c n}} * \text { Basic } \\
& +\beta_{b s, 4} D_{S_{u s, u s}} * \text { Basic }+\beta_{t c, 1} \text { Technology }+\beta_{t c, 2} D_{S_{c n, u s}} * \text { Technology } \\
& +\beta_{t c, 3} D_{S_{u s, c n}} * \text { Technology }+\beta_{t c, 4} D_{S_{u s, u s}} * \text { Technology }+\beta_{f n, 1} \text { Financial } \\
& +\beta_{f n, 2} D_{S_{c n, u s}} * \text { Financial }+\beta_{f n, 3} D_{S_{u s, c n}} * \text { Financial }+\beta_{f n, 4} D_{S_{u s, u s}} * \text { Financial }
\end{aligned}
$$

29 The 115 companies are classified into 8 sectors. There are 70 companies in the basic materials sector, 10 companies in the technology sector, 9 companies in the financial sector, and 26 companies in the other five sectors (consumer goods, heath care, industrial goods, services, and utilities). Eun and Sabherwal (2003) find that the sectors do not affect price discovery significantly. In this research, we reexamine price discovery efficiency across sectors.

30 Eun and Sabherwal (2003) use the trade-by-trade data while we use the minute-by-minute data. Therefore, we use the trading volume variation within a small window, say 1 minute, to get a sense of medium trades. When the variation is small, this is a reflection of less large trades and/or less small trades but more medium trades. 


$$
\begin{aligned}
& +\beta_{\text {alt }, 1} \text { Alternext }+\beta_{\text {alt }, 2} D_{S_{c n, u s}} * \text { Alternext }+\beta_{a l t, 3} D_{S_{u s, c n}} * \text { Alternext } \\
& +\beta_{a l t, 4} D_{S_{u s, u s}} * \text { Alternext }+\beta_{n s d, 1} \text { NASDAQ }+\beta_{n s d, 2} D_{S_{c n, u s}} * \text { NASDAQ } \\
& \quad+\beta_{n s d, 3} D_{S_{u s, c n}} * \text { NASDAQ }+\beta_{n s d, 4} D_{S_{u s, u s}} * \text { NASDAQ }+\varepsilon_{i, j}
\end{aligned}
$$

where $\beta$ 's are parameters, $\varepsilon_{i, j}$ is a random error, and $i, j=c n$, us. With this model, we can use the estimates for $\beta_{c n, k}$ for all $k$ to test the efficient home market hypothesis and use the estimates for $\beta_{b s, k}, \beta_{t c, k}$, and $\beta_{f n, k}$ for all $k$ to test the sector effect hypothesis. We can also use this model to find out the factors affecting price discovery efficiency within and across the two markets.

The estimation results including the Arellano-Bond robust standard errors of the panel data model are presented in Table 10. The estimation results indicate that if a stock is issued by the Canadian company, the value of $\log \left(S_{c n, c n}\right)$ is higher while the value of $\log \left(S_{c n, u s}\right)$ is lower. This implies that price discovery efficiency for Canadian stocks within Canada is higher but price discovery efficiency is lower when innovations transmit from the U.S. to Canada. Therefore, price discovery efficiency in Canada is higher for Canadian stocks. This confirms the efficient home market hypothesis.

(Please insert Table 10 about here)

We find that if a company has a higher market-cap, the value of $\log \left(S_{c n, c n}\right)$ is higher while the values of $\log \left(S_{c n, u s}\right)$ and $\log \left(S_{u s, u s}\right)$ are lower. This means that price discovery efficiency is higher for large-cap stocks if innovations transmit within Canada but is lower for these stocks if innovations transmit from the U.S. to Canada or within the U.S.

The estimation results indicate that medium trades in both Canada and the U.S. do not affect the value of $\log \left(S_{c n, c n}\right)$. Therefore, medium trades in both the Canadian and U.S. markets do not affect price discovery efficiency within Canada. But a higher level of medium trades in the U.S ${ }^{31}$ is associated with higher price discovery efficiency if innovations transmit from the U.S. to Canada.

From the estimation results, we note that the trading volume in Canada has a positive impact on the value of $\log \left(S_{c n, c n}\right)$ but a negative impact on the value of $\log \left(S_{u s, c n}\right)$. The trading volume in the U.S. has a negative impact on the value of $\log \left(S_{c n, c n}\right)$ but a positive impact on the value of $\log \left(S_{u s, c n}\right)$. Therefore, a higher (lower) trading volume in Canada increases (decreases) price discovery efficiency in Canada. But it is not the case for trading volume in the U.S. This explains why the home market efficiency hypothesis is supported in view of the majority of the stocks are Canadian stocks.

We find that the value of $\log \left(S_{c n, c n}\right)$ is higher for stocks in the basic materials sector, but the values of $\log \left(S_{c n, u s}\right)$ and $\log \left(S_{u s, u s}\right)$ are lower for stocks in the same sector. This implies that stocks in the basic materials sector have higher price discovery efficiency in Canada but price discovery efficiency is lower for stocks in the basic materials sector if innovations transmit from the U.S. to Canada and within the U.S. However, stocks in the technology and financial sectors do not change the values of $\log \left(S_{c n, c n}\right), \log \left(S_{c n, u s}\right)$, $\log \left(S_{u s, c n}\right)$, and $\log \left(S_{u s, u s}\right)$. That means that there is no obvious difference in price discovery efficiency for stocks in the technology and financial sectors no matter whether innovations transmit within and across the Canadian and U.S. markets. These findings corroborate the niche role of the Canadian stock exchange as one of the world's major exchanges for stocks in the basic materials sector. The findings support the sector effect hypothesis.

When examining the role of the exchanges, we find that if stocks are traded in the NYSE Alternext, the value of $\log \left(S_{c n, c n}\right)$ is lower, but the value of $\log \left(S_{u s, u s}\right)$ is higher, relative to those traded in the NYSE. These findings corroborate the fact that stocks traded in the NYSE Alternext are primarily junior stocks and are mostly exposed to the U.S. NYSE Alternext. We find that if stocks are traded in the NASDAQ, the value of $\log \left(S_{c n, c n}\right)$ is not affected, but the value of $\log \left(S_{u s, c n}\right)$ is higher, relative to stocks traded in the NYSE. These findings corroborate the fact that stocks traded in the NASDAQ are typically technology stocks and that innovations transmitting from Canada to the U.S. is more efficient in the NASDAQ. Once again, these findings further reinforce the support for the sector effect hypothesis.

\footnotetext{
31 A higher level of medium trades is represented by a smaller standard deviation of the minute-by-minute trading volumes.
} 


\section{Concluding Remarks}

The empirical evidence suggests the underlying structure of price discovery processes is more complex and the standard structural model cannot accommodate market-specific shocks that arrive at different points in time, are of different distributions, and get absorbed within and across different markets with different lags. In view of this, we have extended the standard structural model to accommodate these non-homogeneous and timevarying shocks and, based on the extension, we have proposed a novel methodology to study price discovery processes. This methodology includes a strategy for identifying information channels, a new dynamic price discovery measure, and a panel data model for price discovery efficiency within and across markets at various intraday data frequencies. We use the methodology to study the 115 stocks cross-listed in the Canadian and U.S. stock markets.

Using our information channel identification strategy and intraday data of the 115 stocks traded in the Canadian and U.S. markets, we find that, for the 115 stocks, there are more information channels from Canada to the U.S. than those from the U.S. to Canada across various intraday data frequencies. We have gauged how innovations affect stock prices within and across the two markets over time using the dynamic price discovery measure. We find that the market tends to over-react (under-react) to innovations coming from within (from outside) and that pricing discrepancies would vanish over time.

Using the panel data model, we have analyzed the price discovery efficiency measures within and across the two markets at various intraday data frequencies and tested the efficient home market and sector effect hypotheses. We find the supporting evidence for the efficient home market hypothesis. Price discovery for Canadian stocks is efficient in the Canadian market. This higher efficiency is directly related to a higher trading volume in the Canadian market. This finding explains why the home market for the Canadian stocks is more efficient in price discovery. We also find the supporting evidence for the sector effect hypothesis. Price discovery for stocks in the basic materials sector is more efficient in Canada. This confirms that the Canadian TSX is one of the world's major exchanges specialized in the basic materials sector. In addition, We find that when innovations transmit within or from the U.S., price discovery for junior stocks traded in the NYSE Alternext is more efficient. When innovations transmit from Canada to the U.S., price discovery for technology stocks traded in the NASDAQ is more efficient. These findings not only reflect the specialized role of each exchange but also provide additional support for the sector effect hypothesis.

Acknowledgements Lei Wu thanks the National Natural Science Foundation of China for support (Grant No. 71671). Kuan $\mathrm{Xu}$ thanks University of Toronto and Renmin University of China for providing visiting opportunities during the Winter and Summer of 2018. The authors wish to thank Dr. C.-F. Lee, Editor-in-Chief, Review of Quantitative Finance and Accounting, and two anonymous referees for helpful comments and suggestions. The authors also wish to thank John Galbraith, Yin-Feng Gau, Victoria Zinde-Walsh, Xue Wang, and other participants at the 23rd Conference on the Theory and Practices of Securities and Financial Markets at National Sun Yat-sen University, the Frontiers of Business Research in China 2016 International Symposium, and the Canadian Econometrics Study Group 2017 Meeting for helpful comments on earlier drafts of the paper. Any remaining errors are of our own. 


\section{References}

1. Adams G, McQueen G, Wood R (2004) The effects of inflation news on high frequency stock returns. Journal of Business 77(3):547-574. https://doi.org/10.1086/386530

2. Agarwal S, Liu C, Rhee S G (2007) Where does price discovery occur for stocks traded in multiple markets? Evidence from Hong Kong and London. Journal of International Money and Finance 26(1):46-63. https: //doi.org/10.1016/j.jimonfin.2006.10.011

3. Bacidore J M, Sofianos G (2002) Liquidity provision and specialist trading in NYSE-listed non-U.S. stocks. Journal of Financial Economics 63(1):133-158. https://doi.org/10.1016/S0304-405X(01)00092-7

4. Booth G G, So R W, Tse Y (1999) Price discovery in the German equity index derivatives markets. Journal of Futures Markets 19(6):619-643 https://doi.org/10.1002/(SICI) 1096-9934(199909) 19:6<619: :AID-FUT1>3. $0 . \mathrm{CO} ; 2-\mathrm{M}$

5. Brogaard J, Hendershott T, Riordan R (2014) High-frequency trading and price discovery. Review of Finacial Studies 27(8):2267-2306. https://doi.org/10.1093/rfs/hhu032

6. Brooks R, Patel A, Su T (2003) How the equity market responds to unanticipated events. Journal of Business 76(1):109-133. https://doi.org/10.1086/344115

7. Chaboud A P, Chiquoine B, Hjalmarsson E, Vega C (2014) Rise of the machines: Algorithmic trading in the foreign exchange market. Journal of Finance 69(5):2045-2084. https://doi.org/10.1111/jofi.12186

8. Chang T K (1998) All countries not created equal to be news: World system and international communication. Communication Research 25(5):528-563. https://doi.org/10.1177/009365098025005004

9. Chiang R, Fong W-M (2001) Relative informational effiency of cash, futures, and options markets: The case of an emerging market. Journal of Banking and Finance 25(2):355-375. https://doi.org/10.1016/S03784266(99) 00127-2

10. Chu Q C, Hsieh W G, Tse Y (1999) Price discovery on the S\&P 500 index markets: An analysis of spot index, index futures, and SPDRs. International Review of Financial Analysis 8(1):21-34. https://doi.org/ 10.1016/S1057-5219(99)00003-4

11. Ellul, A (2006) Ripples through markets: Inter-market impacts generated by large trades. Journal of Financial Economics 82(1):173-196. https://doi.org/10.1016/j.jfineco.2005.05.011

12. Eun C S, Sabherwal S (2003) Cross-border listings and price discovery: Evidence from U.S.-listed Canadian stocks. Journal of Finance 58(2):459-575. https://doi.org/10.1111/1540-6261.00537

13. Frijns B, Gilbert A, Tourani-Rad A (2015a) The determinants of price discovery: Evidence from US-Canada cross-listed shares. Journal of Banking and Finance 59:457-468. https://doi.org/10.1016/j ·jbankfin.2015. 07.011

14. Frijns B, Indriawan I, Tourani-Rad A (2015b) Macroeconomic news announcements and price discovery: Evidence from Canadian-U.S. cross-listed firms. Journal of Empirical Finance 32:35-48. https://doi.org/ $10.1016 / j \cdot j$ empfin.2014.05.001

15. Frijns B, Indriawan I, Tourani-Rad A (2018) The intractions between price discovery, liquidity and algorithmic trading for U.S.-Canadian cross-listed shares, International Review of Financial Analysis 56:136-152. https://doi.org/10.1016/j.irfa.2018.01.005

16. Frijns B, Schotman P (2009) Price discovery in tick time. Journal of Empirical Finance 16(5):759-776. https://doi.org/10.1016/j.jempfin.2009.07.002

17. Gonzalo J, Granger C (1995) Estimation of common long-memory components in cointegration systems. Journal of Business \& Economic Statistics 13(1):27-35. https://doi.org/10.1080/07350015.1995.10524576

18. Grammig J, Melvin M, Schlag C (2005) Internationally cross-listed stock prices during overlapping trading hours: Price discovery and exchange rate effects. Journal of Empirical Finance 12(1):139-164. https://doi. org/10.1016/j.jempfin.2003.10.004

19. Harris F H deB, McInish T H, Wood R A (2002) Security price adjustment across exchanges: An investigation of common factor components in Dow stocks. Journal of Financial Markets 5(3):277-308. https://doi.org/10.1016/S1386-4181(01)00017-9

20. Hasbrouck J (1995) One security, many markets: Determining the contributions to price discovery. Journal of Finance 50(4):1175-1199. https://doi.org/10.1111/j.1540-6261.1995.tb04054.x

21. Hasbrouck J (2003) Intraday price formation in US equity index markets. Journal of Finance 58(6):23752400. https://doi.org/10.1046/j.1540-6261.2003.00609.x

22. Hatheway F, Kwan A, Zheng H (2017) An empirical analysis of market segmentation on U.S. equity markets. Journal of Financial and Quantitative Analysis 52(6):2399-2427. https://doi.org/10.1017/ 


\section{S0022109017000849}

23. Hautsch N, Hess D, Veredas D (2011) The impact of macroeconomic news on quote adjustments, noise, and informational volatility. Journal of Banking and Finance 35(10):2733-2746. https://doi.org/10.1016/ j.jbankfin.2011.03.004

24. Heinkel R, Kraus A (1988) Measuring event impacts in thinly traded stocks. Journal of Financial and Quantitative Analysis 23(1):71-88. https://doi.org/10.2307/2331025

25. Hupperets E C J, Menkveld and A J (2002) Intraday analysis of market integration: Dutch blue chips traded in Amsterdam and New York. Journal of Financial Markets 5(1):57-82. https://doi.org/10.1016/S13864181(01) 00019-2

26. Johansen S (1991) Cointegration and hypothesis testing of cointegration vectors in Gaussian vector autoregressive models. Econometrica 59:1551-1580. https://doi.org/10.2307/2938278

27. Kryzanowski L, Zhang H (2002) Intraday price integration for shares cross-listed internationally. Journal of Financial and Quantitative Analysis 37(2):243-269. https://doi.org/10.2307/3595005

28. Lee C-C., Chen M-P, Chang C-H (2014) Industry co-movement and cross-listing: Do home country factors matter?, Japan and the World Economy 32:96-110. https://doi.org/10.1016/j.japwor.2014.09.001

29. Menkveld A J (2008) Splitting orders in overlapping markets: a study of cross-listed stocks. Journal of Financial Intermediation 17(2):145-174. https://doi.org/10.1016/j.jfi.2007.05.004

30. Menkveld A J, Zhou Y B, Zhu H (2017) Shades of darkness: A pecking order of trading venues. Journal of Financial Economics 124(3):503-534. https://doi.org/10.1016/j.jfineco.2017.03.004

31. Otsubo Y (2014) International cross-listing and price discovery under trading concentration in the domestic market: Evidence from Japanese shares. Journal of Empirical Finance 25:36-51. https://doi.org/10.1016/ j.jempfin.2013.11.003

32. Putniņš T J (2013) What do price discovery metrics really measure? Journal of Empirical Finance 23:68-83. https://doi.org/10.1016/j.jempfin.2013.05.004

33. Securities and Exchange Commission (2010) Concept release on equity market structure. Release No. 3451358. File No. 57-2-10.

34. Solnik B H (1996) International investments. Addison-Wesley, Reading, M.A.

35. Vives X (1993) How fast do rational agents learn? Review of Economic Studies 60(2):329-347. https: //doi.org/10.2307/2298060

36. Vives X (1997) Learn from others: A welfare analysis. Games and Economic Behavior 20(2):177-200. https://doi.org/10.1006/game.1997.0562

37. Wang Q, Yang H (2015) Earnings announcements, trading volume, and price discovery: evidence from dual class firms. Review of Quantitative Finanance and Accounting 44(4):669-700. https://doi.org/10.1007/ s11156-013-0422-4

38. Werner I M, Kleidon A (1996) U.K. and U.S. trading of British cross-listed stocks: an intraday analysis of market integration. The Review of Financial Studies 9(2):619-664. https://doi.org/10.1093/rfs/9.2.619

39. Wu L, Meng Q, Xu K (2015) 'Slow-burn' spillover and 'fast and furious' contagion: a study of international stock markets. Quantitative Finance 15(6):933-958. https://doi.org/10.1080/14697688.2014.952242

40. Yan B, Zivot E (2010) A structural analysis of price discovery measure. Journal of Financial Markets 13(1):1-19. https://doi.org/10.1016/j.finmar.2009.09.003

41. Yeh Y-H, Shu P-G, Yang Y-W (2016) How insiders' personal incentives and timeliness of information revelation are related to their sales timing. Review of Pacific Basin Financial Markets and Policies 19(2):1650009. https://doi.org/10.1142/S0219091516500090 
Fig. $1 \frac{\left|\alpha_{c n}\right|}{\left|\alpha_{u s}\right|}$ for Intraday Data of 60-minute Frequency as Initial Sampling Time Shifts Minute by Minute

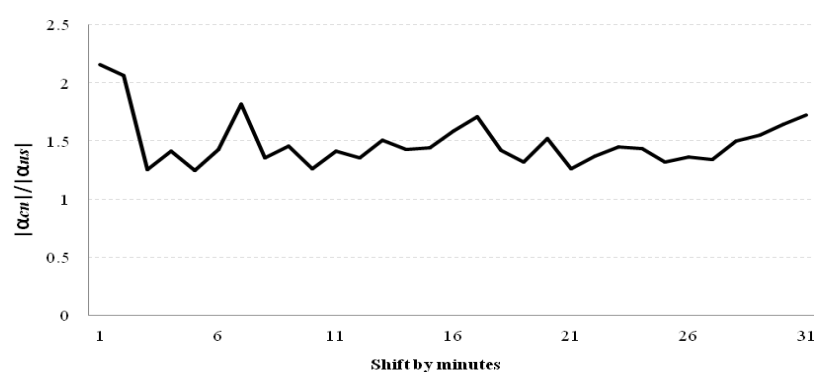

Notes: Based on the intraday data of the 60-minute frequency, when the sampling time shifts by 1 minute a time, $\frac{\left|\alpha_{c n}\right|}{\left|\alpha_{u s}\right|}$ is always greater than 1 at all shifts. 
Fig. $2 \frac{\left|\alpha_{c n}\right|}{\left|\alpha_{u s}\right|}$ for Intraday Data of 30-minute Frequency as Initial Sampling Time Shifts Minute by Minute

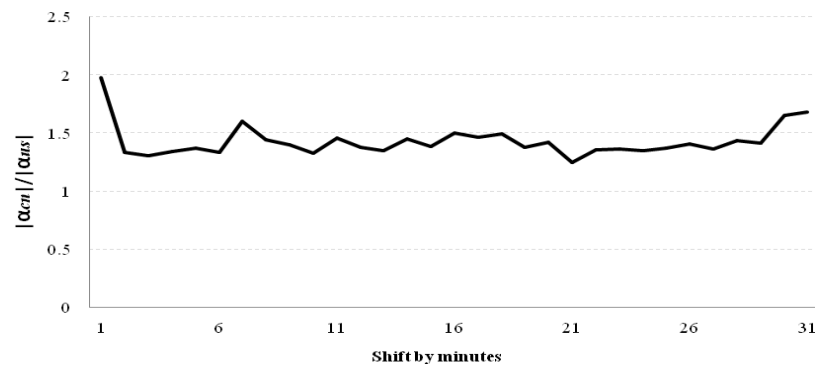

Notes: Based on the intraday data of the 30-minute frequency, when the sampling time shifts by 1 minute a time, $\frac{\left|\alpha_{c n}\right|}{\left|\alpha_{u s}\right|}$ is always greater than 1 at all shifts. 
Fig. $3 \frac{\left|\alpha_{c n}\right|}{\left|\alpha_{u s}\right|}$ for Intraday Data of 10-minute Frequency as Initial Sampling Time Shifts Minute by Minute

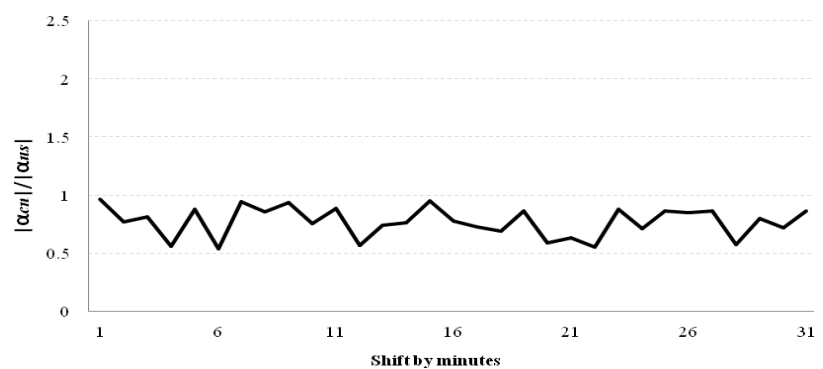

Notes: Based on the intraday data of the 10-minute frequency, when the initial sampling time shifts by 1 minute a time, $\frac{\left|\alpha_{c n}\right|}{\left|\alpha_{u s}\right|}$ is less than 1 at all shifts. 
Fig. $4 \frac{\left|\alpha_{c n}\right|}{\left|\alpha_{u s}\right|}$ for Intraday Data of 5-minute Frequency as Initial Sampling Time Shifts Minute by Minute

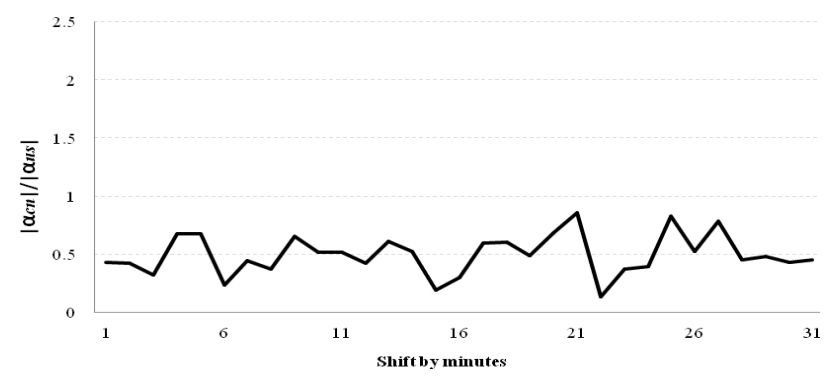

Notes: Based on the intraday data of the 5-minute frequency, when the initial sampling time shifts by 1 minute a time, $\frac{\left|\alpha_{c n}\right|}{\left|\alpha_{u s}\right|}$ is always less than 1 at all shifts. 
Fig. 5 Dynamics of Barrick Gold (ABX)'s $D_{u s, c n, t}$ Based on Intraday Data of 10-minute Frequency

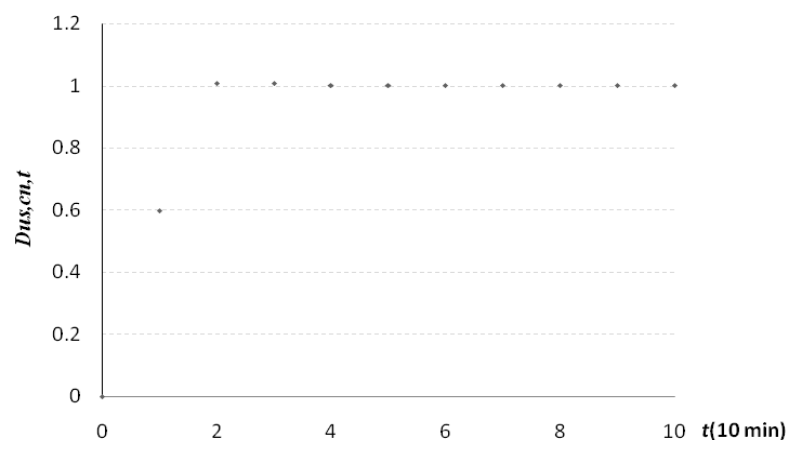

Notes: The price discovery measure converges to 1 at about 20 minutes $(t=2)$. 
Fig. 6 Dynamics of Barrick Gold (ABX)'s $D_{u s, c n, t}$ Based on Intraday Data of 5-minute Frequency

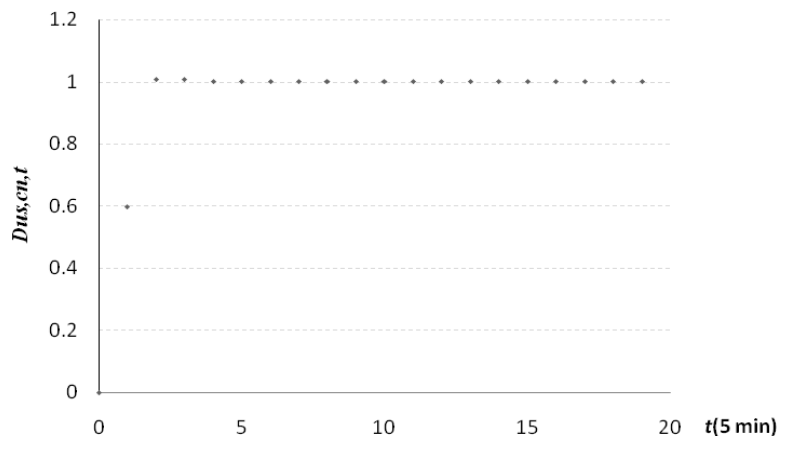

Notes: The price discovery measure converges to 1 at about 10-20 minutes $(t=2 \sim 3)$. 
Fig. 7 Dynamics of Barrick Gold (ABX)'s $D_{u s, c n, t}$ Based on Intraday Data of 1-minute Frequency

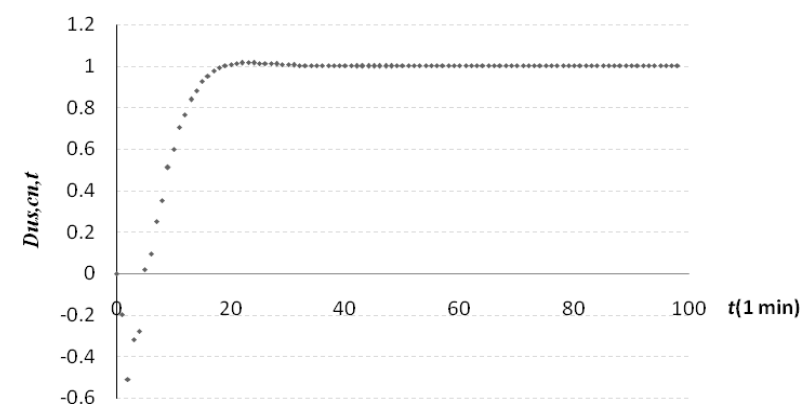

Notes: The price discovery measure converges to 1 at about 20 minutes $(t=20)$. 
Table 1 Basic Information of Companies Actively Traded in the U.S. and Canadian Stock Exchanges: Feb. 14, 2016-July. 28, 2017

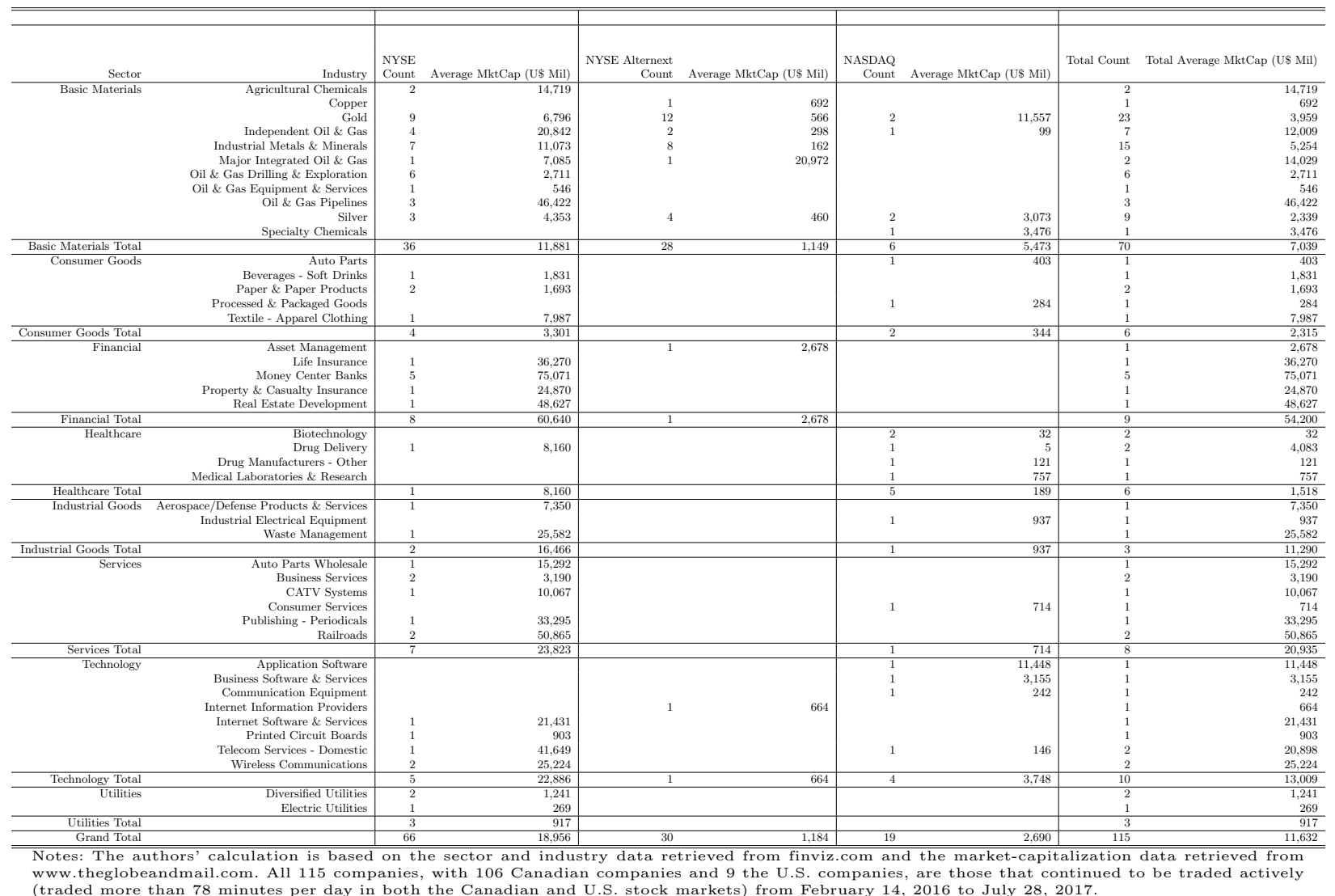


Table 2 Cointegration Analysis

\begin{tabular}{|c|c|c|c|c|c|c|c|c|}
\hline & $1 \mathrm{emin}$ & $5 \min$ & $10 \mathrm{~min}$ & $15 \mathrm{~min}$ & $30 \mathrm{~min}$ & $65 \mathrm{~min}$ & 1 day close & 1 day open \\
\hline & -1.003 & -1.003 & -1.003 & -1.001 & -1.002 & -1 & -0.981 & -1.001 \\
\hline$\beta_{u s}$ & $(-1.021,-0.956)$ & $(-1.021,-0.957)$ & $(-1.022,-0.956)$ & $(-1.022,-0.956)$ & $(-1.022,-0.959)$ & $(-1.023,-0.961)$ & $(-1.061,-0.802)$ & $(-1.014,-0.995)$ \\
\hline \multirow[b]{2}{*}{$\beta_{i d c n}$} & 0 & 0 & 0 & 0 & 0 & 0 & -0.011 & 0.003 \\
\hline & $(-0.084,0.032)$ & $(-0.082,0.035)$ & $(-0.081,0.034)$ & $(-0.082,0.000)$ & $(-0.077,0.009)$ & $(-0.080,0.000)$ & $(-0.136,0.162)$ & $(-0.012,0.011)$ \\
\hline \multirow[b]{2}{*}{$\beta_{i d u s}$} & 0 & 0 & 0 & 0 & 0.001 & 0.002 & -0.006 & -0.003 \\
\hline & $(-0.015,0.041)$ & $(-0.012,0.042)$ & $(-0.003,0.042)$ & $(0.000,0.042)$ & $(0.000,0.043)$ & $(0.000,0.045)$ & $(-0.088,0.027)$ & $(-0.008,0.006)$ \\
\hline \multirow[b]{2}{*}{$\alpha_{c n}$} & -0.003 & -0.006 & -0.01 & -0.048 & -0.07 & -0.099 & -0.173 & -0.378 \\
\hline & $(-0.023,0.000)$ & $(-0.086,0.002)$ & $(-0.137,0.003)$ & $(-0.177,0.004)$ & $(-0.288,0.009)$ & $(-0.434,0.029)$ & $(-0.681,0.078)$ & $(-0.777,-0.019)$ \\
\hline \multirow{3}{*}{$\alpha_{u s}$} & 0.005 & 0.013 & 0.023 & 0.042 & 0.071 & 0.124 & 0.267 & 0.466 \\
\hline & $(0.001,0.026)$ & $(0.003,0.056)$ & $(0.005,0.088)$ & $(0.008,0.115)$ & $(0.013,0.163)$ & $(0.023,0.263)$ & $(-0.057,0.879)$ & $(0.000,0.811)$ \\
\hline & 0 & 0 & 0 & 0 & 0 & 0 & -0.014 & -0.065 \\
\hline \multirow{2}{*}{$\alpha_{i d c n}$} & $(0.000,0.000)$ & $(0.000,0.000)$ & $(0.000,0.000)$ & $(0.000,0.002)$ & $(0.000,0.002)$ & $(0.000,0.005)$ & $(-0.111,0.000)$ & $(-0.290 .0 .017)$ \\
\hline & 0 & 0 & 0.002 & 0.003 & 0.005 & 0.01 & 0.037 & 0.043 \\
\hline$\alpha_{i d u s}$ & $(0.000,0.001)$ & $(0.000,0.003)$ & $(0.000,0.006)$ & $(0.000,0.009)$ & $(0.000,0.017)$ & $(0.000,0.028)$ & $(0.000,0.173)$ & $(0.000,0.262)$ \\
\hline \multirow{2}{*}{$\left|\alpha_{c n} / \alpha_{u s}\right|$} & 0.65 & 0.584 & 0.845 & 1.438 & 1.857 & 1.877 & 3.203 & 8.919 \\
\hline & $(0.116,4,510)$ & $(0.076,7.346)$ & $(0.049,7.359)$ & $(0.270,7.264)$ & $(0.390,6.903)$ & $(0.412,7.357)$ & $(0.305,10.422)$ & $(0.583,14,735)$ \\
\hline
\end{tabular}

Table 3 Alternative Specifications of $\mathbf{A}$

\begin{tabular}{|c|c|c|c|c|c|}
\hline $\mathbf{A}_{1}=$ & {$\left[\begin{array}{cccc}1 & N a & N a & 0 \\
0 & 1 & N a & N a \\
0 & 0 & 1 & N a \\
0 & 0 & 0 & 1\end{array}\right]$} & $\mathbf{A}_{2}=$ & $\left.\begin{array}{cccc}1 & N a & N a & 0 \\
0 & 1 & N a & N a \\
0 & 0 & 1 & 0 \\
0 & 0 & N a & 1\end{array}\right]$ & $\mathbf{A}_{3}=$ & $\left.\begin{array}{cccc}1 & 0 & N a & 0 \\
N a & 1 & N a & N a \\
0 & 0 & 1 & N a \\
0 & 0 & 0 & 1\end{array}\right]$ \\
\hline $\mathbf{A}_{4}=$ & {$\left[\begin{array}{cccc}1 & 0 & N a & 0 \\
N a & 1 & N a & N a \\
0 & 0 & 1 & 0 \\
0 & 0 & N a & 1\end{array}\right]$} & $\mathbf{A}_{5}=$ & {$\left[\begin{array}{cccc}1 & N a & 0 & N a \\
0 & 1 & N a & N a \\
0 & 0 & 1 & N a \\
0 & 0 & 0 & 1\end{array}\right]$} & $\mathbf{A}_{6}=$ & {$\left[\begin{array}{cccc}1 & N a & 0 & N a \\
0 & 1 & N a & N a \\
0 & 0 & 1 & 0 \\
0 & 0 & N a & 1\end{array}\right]$} \\
\hline $\mathbf{A}_{7}=$ & {$\left[\begin{array}{cccc}1 & 0 & 0 & N a \\
N a & 1 & N a & N a \\
0 & 0 & 1 & N a \\
0 & 0 & 0 & 1\end{array}\right]$} & $\mathbf{A}_{8}=$ & $\left.\begin{array}{cccc}1 & 0 & 0 & N a \\
N a & 1 & N a & N a \\
0 & 0 & 1 & 0 \\
0 & 0 & N a & 1\end{array}\right]$ & $\mathbf{A}_{9}=$ & $\left.\begin{array}{cccc}1 & N a & N a & N a \\
0 & 1 & 0 & N a \\
0 & 0 & 1 & N a \\
0 & 0 & 0 & 1\end{array}\right]$ \\
\hline $\mathbf{A}_{10}=$ & {$\left[\begin{array}{cccc}1 & N a & N a & N a \\
0 & 1 & 0 & N a \\
0 & 0 & 1 & 0 \\
0 & 0 & N a & 1\end{array}\right]$} & $\mathbf{A}_{11}=$ & {$\left[\begin{array}{cccc}1 & 0 & N a & N a \\
N a & 1 & 0 & N a \\
0 & 0 & 1 & N a \\
0 & 0 & 0 & 1\end{array}\right]$} & $\mathbf{A}_{12}=$ & $\left.\begin{array}{cccc}1 & 0 & N a & N a \\
N a & 1 & 0 & N a \\
0 & 0 & 1 & 0 \\
0 & 0 & N a & 1\end{array}\right]$ \\
\hline $\mathbf{A}_{13}=$ & {$\left[\begin{array}{cccc}1 & N a & N a & N a \\
0 & 1 & N a & 0 \\
0 & 0 & 1 & N a \\
0 & 0 & 0 & 1\end{array}\right]$} & $\mathbf{A}_{14}=$ & {$\left[\begin{array}{cccc}1 & N a & N a & N a \\
0 & 1 & N a & 0 \\
0 & 0 & 1 & 0 \\
0 & 0 & N a & 1\end{array}\right]$} & $\mathbf{A}_{15}=$ & {$\left[\begin{array}{cccc}1 & 0 & N a & N a \\
N a & 1 & N a & 0 \\
0 & 0 & 1 & N a \\
0 & 0 & 0 & 1\end{array}\right]$} \\
\hline & & $\mathbf{A}_{16}=$ & {$\left[\begin{array}{cccc}1 & 0 & N a & N a \\
N a & 1 & N a & 0 \\
0 & 0 & 1 & 0 \\
0 & 0 & N a & 1\end{array}\right]$} & & \\
\hline
\end{tabular}

$\overline{\text { Notes: } 0 \text { and } 1 \text { are } 0 \text { and } 1 \text { restrictions, respectively. "Na" is the free parameter }}$ with no restriction imposed. 
Table 4 Numbers of Stocks Satisfying a Specific Over-identified $A$ Based on LR Tests

\begin{tabular}{ccccccc}
\hline \hline A specification & $1 \mathrm{~min}$ & $5 \mathrm{~min}$ & $10 \mathrm{~min}$ & $15 \mathrm{~min}$ & $30 \mathrm{~min}$ & $65 \mathrm{~min}$ \\
\hline $\mathbf{A}_{1}$ & 20 & 11 & 10 & 10 & 8 & 6 \\
$\mathbf{A}_{3}$ & 17 & 25 & 27 & 24 & 25 & 25 \\
$\mathbf{A}_{5}$ & 11 & 13 & 10 & 13 & 11 & 10 \\
$\mathbf{A}_{7}$ & 4 & 6 & 4 & 4 & 3 & 4 \\
$\mathbf{A}_{9}$ & 19 & 16 & 12 & 16 & 15 & 17 \\
$\mathbf{A}_{11}$ & 34 & 35 & 35 & 29 & 35 & 35 \\
$\mathbf{A}_{13}$ & 7 & 7 & 13 & 14 & 16 & 14 \\
$\mathbf{A}_{15}$ & 3 & 2 & 4 & 5 & 2 & 4 \\
\hline From Canada to the U.S. & 58 & 69 & 70 & 62 & 65 & 68 \\
From the U.S. to Canada & 57 & 46 & 45 & 53 & 50 & 47 \\
\hline \hline
\end{tabular}

Notes: This table presents the results on the number of stocks sharing the information channels given by $\mathbf{A}_{i}$ at a certain frequency. Our testing results indicate that no stock based on the intraday data of various frequencies satisfies the patterns of information channels embedded in $\mathbf{A}_{2}, \mathbf{A}_{4}, \mathbf{A}_{6}, \mathbf{A}_{8}$, $\mathbf{A}_{10}, \mathbf{A}_{12}, \mathbf{A}_{14}$, and $\mathbf{A}_{16}$. However, the other patterns of information channels embedded in $\mathbf{A}_{1}, \mathbf{A}_{3}$, $\mathbf{A}_{5}, \mathbf{A}_{7}, \mathbf{A}_{9}, \mathbf{A}_{11}, \mathbf{A}_{13}$, and $\mathbf{A}_{15}$ are identified for some, but not all, stocks at some frequencies. The lower panel of this table summarizes the total numbers of two key types of information channels"from Canada to the U.S." and "from the U.S. to Canada" and shows that there are more information channels from Canada to the U.S. rather than from the U.S. to Canada. The interpretation is that the Canada market releases more information than the U.S. market about these stocks, the overwhelming majority of which are issued by Canadian corporations. 
Table 5 Information Transmission at 1, 5-, and 10-minute Frequencies

\begin{tabular}{|c|c|c|c|c|c|}
\hline \multicolumn{6}{|c|}{ Panel A: 1 min data } \\
\hline & $t=0$ & $t=50$ & $t=100$ & $t=150$ & $t=200$ \\
\hline$D_{c n, c n, t}$ & 1.859 & 1.302 & 1.153 & 1.078 & 1.037 \\
\hline std. error & 3.540 & 1.680 & 0.879 & 0.442 & 0.203 \\
\hline$D_{u s, c n, t}$ & 0.355 & 0.833 & 0.903 & 0.951 & 0.967 \\
\hline std. error & 0.421 & 0.297 & 0.225 & 0.163 & 0.105 \\
\hline$D_{c n, u s, t}$ & 0.412 & 0.908 & 0.941 & 0.946 & 0.975 \\
\hline std. error & 0.430 & 0.569 & 0.305 & 0.161 & 0.080 \\
\hline$D_{u s, u s, t}$ & 1.876 & 1.205 & 1.124 & 1.043 & 1.003 \\
\hline std. error & 0.887 & 0.320 & 0.178 & 0.104 & 0.058 \\
\hline \multicolumn{6}{|c|}{ Panel B: 5 min data } \\
\hline & $t=0$ & $t=10$ & $t=20$ & $t=30$ & $t=40$ \\
\hline$D_{c n, c n, t}$ & 2.181 & 1.526 & 1.274 & 1.140 & 1.038 \\
\hline std. error & 5.323 & 2.782 & 1.418 & 0.697 & 0.108 \\
\hline$D_{u s, c n, t}$ & 0.319 & 0.827 & 0.888 & 0.928 & 0.958 \\
\hline std. error & 0.420 & 1.975 & 1.549 & 1.141 & 0.747 \\
\hline$D_{c n, u s, t}$ & 0.551 & 0.838 & 0.899 & 0.938 & 0.965 \\
\hline std. error & 0.466 & 0.422 & 0.231 & 0.130 & 0.071 \\
\hline$D_{u s, u s, t}$ & 1.760 & 1.222 & 1.156 & 1.040 & 1.021 \\
\hline std. error & 0.725 & 0.278 & 0.192 & 0.128 & 0.238 \\
\hline \multicolumn{6}{|c|}{ Panel C: 10 min data } \\
\hline & $t=0$ & $t=5$ & $t=10$ & $t=15$ & $t=20$ \\
\hline$D_{c n, c n, t}$ & 1.847 & 1.299 & 1.156 & 1.085 & 1.041 \\
\hline std. error & 2.892 & 1.069 & 0.512 & 0.296 & 0.143 \\
\hline$D_{u s, c n, t}$ & 0.333 & 0.795 & 0.915 & 0.958 & 0.970 \\
\hline std. error & 0.440 & 2.189 & 1.710 & 1.254 & 0.819 \\
\hline$D_{c n, u s, t}$ & 0.576 & 0.867 & 0.907 & 0.939 & 0.963 \\
\hline std. error & 0.470 & 0.212 & 0.142 & 0.094 & 0.059 \\
\hline$D_{u s, u s, t}$ & 1.665 & 1.220 & 1.156 & 1.107 & 1.040 \\
\hline std. error & 0.651 & 0.291 & 0.200 & 0.135 & 0.245 \\
\hline
\end{tabular}

Notes: $t$ is the time lag for the intraday data at a certain frequency. In order to convert a particular time lag $t$ into the number of minutes for the intraday data, please multiply time lag $t$ by the intraday data frequency in minutes. $D_{i, j, t}$ is the price discovery measure for the impact of innovations in market $j$ on market $i$. The price discovery measure is the average of all such measures for the 115 stocks. The number below $D_{i, j, t}$ is the standard error. The numbers in bold font are those price discovery measures that first reach the range of $1 \pm 0.05$ as time lag $t$ increases. When $D_{i, j, t}$ approaches 1 , this indicates that there is no pricing error in market $i$ caused by market $j$ at time lag $t$. 
Table 6 Information Transmission at 15-, 30-, and 65-minute Frequencies

\begin{tabular}{|c|c|c|c|c|c|}
\hline \multicolumn{6}{|c|}{ Panel D: 15 min data } \\
\hline & $t=0$ & $t=4$ & $t=8$ & $t=12$ & $t=16$ \\
\hline$D_{c n, c n, t}$ & 1.990 & 1.303 & 1.148 & 1.024 & 1.012 \\
\hline std. error & 4.435 & 1.279 & 0.589 & 0.272 & 0.094 \\
\hline$D_{u s, c n, t}$ & 0.414 & 0.752 & 0.897 & 0.969 & 0.991 \\
\hline std. error & 0.462 & 1.186 & 0.859 & 0.551 & 0.226 \\
\hline$D_{c n, u s, t}$ & 0.509 & 0.900 & 0.917 & 0.976 & 0.996 \\
\hline std. error & 0.482 & 0.470 & 0.214 & 0.103 & 0.043 \\
\hline$D_{u s, u s, t}$ & 1.537 & 1.167 & 1.116 & 1.033 & 1.012 \\
\hline std. error & 0.578 & 0.265 & 0.174 & 0.104 & 0.048 \\
\hline \multicolumn{6}{|c|}{ Panel E: 30 min data } \\
\hline & $t=0$ & $t=2$ & $t=4$ & $t=6$ & $t=8$ \\
\hline$D_{c n, c n, t}$ & 1.443 & 1.137 & 1.091 & 1.037 & 1.015 \\
\hline std. error & 3.183 & 1.724 & 0.517 & 0.341 & 0.134 \\
\hline$D_{u s, c n, t}$ & 0.399 & 0.569 & 0.953 & 0.962 & 0.984 \\
\hline std. error & 0.466 & 0.851 & 0.516 & 0.285 & 0.157 \\
\hline$D_{c n, u s, t}$ & 0.545 & 1.170 & 0.937 & 0.977 & 0.993 \\
\hline std. error & 0.484 & 0.288 & 0.278 & 0.523 & 0.156 \\
\hline$D_{u s, u s, t}$ & 1.522 & 1.166 & 1.022 & 1.009 & 1.001 \\
\hline std. error & 0.737 & 0.325 & 0.259 & 0.148 & 0.068 \\
\hline \multicolumn{6}{|c|}{ Panel F: 65 min data } \\
\hline & $t=0$ & $t=1$ & $t=2$ & $t=3$ & $t=4$ \\
\hline$D_{c n, c n, t}$ & 1.368 & 1.131 & 1.130 & 1.040 & 1.000 \\
\hline std. error & 0.918 & 1.156 & 1.496 & 0.112 & 0.134 \\
\hline$D_{u s, c n, t}$ & 0.382 & 0.706 & 0.889 & 0.987 & 1.000 \\
\hline std. error & 0.467 & 0.767 & 0.601 & 0.273 & 0.155 \\
\hline$D_{c n, u s, t}$ & 0.562 & 0.880 & 0.892 & 0.971 & 0.991 \\
\hline std. error & 0.472 & 0.416 & 0.195 & 0.106 & 0.050 \\
\hline$D_{u s, u s, t}$ & 1.593 & 1.151 & 1.146 & 1.044 & 1.024 \\
\hline std. error & 0.836 & 0.335 & 0.270 & 0.169 & 0.077 \\
\hline
\end{tabular}

Notes: $t$ is the time lag for the intraday data at a certain frequency. In order to convert a particular time lag $t$ into the number of minutes for the intraday data, please multiply time lag $t$ by the intraday data frequency in minutes. $D_{i, j, t}$ is the price discovery measure for the impact of innovations in market $j$ on market $i$. The price discovery measure is the average of all such measures for the 115 stocks. The number below $D_{i, j, t}$ is the standard error. The numbers in bold font are those price discovery measures that first reach the range of $1 \pm 0.05$ as time lag $t$ increases. When $D_{i, j, t}$ approaches 1 , this indicates that there is no pricing error in market $i$ caused by market $j$ at time lag $t$. 
Table 7 Summary of Price Discovery Efficiency Measures

\begin{tabular}{ccccc}
\hline \hline Freq. & $S_{c n, c n}$ & $S_{c n, u s}$ & $S_{u s, c n}$ & $S_{u s, u s}$ \\
\hline $1 \mathrm{~min}$ & $\mathbf{8 3 . 1 8 1}$ & 44.888 & $\mathbf{5 2 . 1 7 2}$ & 36.655 \\
$5 \mathrm{~min}$ & $\mathbf{1 4 1 . 4 7 7}$ & 36.998 & $\mathbf{7 9 . 8 9 2}$ & 36.942 \\
$10 \mathrm{~min}$ & $\mathbf{1 2 3 . 9 7 1}$ & 43.105 & $\mathbf{1 2 1 . 5 8 3}$ & 29.871 \\
$15 \mathrm{~min}$ & $\mathbf{1 7 7 . 0 7 0}$ & 66.499 & $\mathbf{9 7 . 5 3 0}$ & 40.606 \\
$30 \mathrm{~min}$ & $\mathbf{1 4 6 . 6 0 5}$ & 94.762 & $\mathbf{1 0 3 . 9 6 1}$ & 43.092 \\
$65 \mathrm{~min}$ & $\mathbf{1 7 8 . 6 5 8}$ & 107.222 & $\mathbf{1 2 2 . 4 2 9}$ & 55.869 \\
\hline
\end{tabular}

Notes: $S_{i, j}$ is the price discovery efficiency measure for $D_{i, j, t}$ over $t$. The interpretation of $S_{i, j}$ is that the greater (smaller) the value for $S_{i, j}$, the more (less) efficiently the innovations transmit from market $j$ to market $i$, and the more (less) quickly pricing discrepancies vanish in response to shocks from market $j$. Here, $i, j=c n$, us. The columns for $S_{c n, c n}$ and $S_{u s, c n}$ indicate that the information efficiency is the highest within the Canadian market $\left(S_{c n, c n}\right)$ and that it is the second highest from the Canadian market to the U.S. market $\left(S_{u s, c n}\right)$. 
Table 8 Correlations among of Price Discovery Efficiency Measures

\begin{tabular}{|c|c|c|c|c|}
\hline \multicolumn{5}{|c|}{ Panel A: 1 min data } \\
\hline & $S_{c n, c n}$ & $S_{c n, u s}$ & $S_{u s, c n}$ & $S_{u s, u s}$ \\
\hline$S_{c n, c n}$ & 1 & & & \\
\hline$S_{c n, u s}$ & -0.058 & 1 & & \\
\hline$S_{u s, c n}$ & 0.796 & -0.172 & 1 & \\
\hline$S_{u s, u s}$ & -0.156 & 0.501 & -0.196 & 1 \\
\hline \multicolumn{5}{|c|}{ "Panel B: 5 min data } \\
\hline & $S_{c n, c n}$ & $S_{c n, u s}$ & $S_{u s, c n}$ & $S_{u s, u s}$ \\
\hline$S_{c n, c n}$ & 1 & & & \\
\hline$S_{c n, u s}$ & -0.08 & 1 & & \\
\hline$S_{u s, c n}$ & 0.642 & -0.194 & 1 & \\
\hline$S_{u s, u s}$ & -0.152 & 0.331 & -0.228 & 1 \\
\hline \multicolumn{5}{|c|}{ Panel C: 10 min data } \\
\hline & $S_{c n, c n}$ & $S_{c n, u s}$ & $S_{u s, c n}$ & $S_{u s, u s}$ \\
\hline$S_{c n, c n}$ & 1 & & & \\
\hline$S_{c n, u s}$ & -0.145 & 1 & & \\
\hline$S_{u s, c n}$ & 0.239 & -0.143 & 1 & \\
\hline$S_{u s, u s}$ & -0.340 & 0.436 & 0.050 & 1 \\
\hline \multicolumn{5}{|c|}{ Panel D: 15 min data } \\
\hline & $S_{c n, c n}$ & $S_{c n, u s}$ & $S_{u s, c n}$ & $S_{u s, u s}$ \\
\hline$S_{c n, c n}$ & 1 & & & \\
\hline$S_{c n, u s}$ & -0.069 & 1 & & \\
\hline$S_{u s, c n}$ & 0.899 & -0.152 & 1 & \\
\hline$S_{u s, u s}$ & -0.105 & 0.155 & -0.128 & 1 \\
\hline \multicolumn{5}{|c|}{ Panel E: 30 min data } \\
\hline & $S_{c n, c n}$ & $S_{c n, u s}$ & $S_{u s, c n}$ & $S_{u s, u s}$ \\
\hline$S_{c n, c n}$ & 1 & & & \\
\hline$S_{c n, u s}$ & -0.116 & 1 & & \\
\hline$S_{u s, c n}$ & 0.539 & -0.246 & 1 & \\
\hline$S_{u s, u s}$ & -0.263 & 0.284 & -0.248 & 1 \\
\hline \multicolumn{5}{|c|}{ Panel F: 65 min data } \\
\hline & $S_{c n, c n}$ & $S_{c n, u s}$ & $S_{u s, c n}$ & $S_{u s, u s}$ \\
\hline$S_{c n, c n}$ & 1 & & & \\
\hline$S_{c n, u s}$ & -0.167 & 1 & & \\
\hline$S_{u s, c n}$ & 0.792 & -0.291 & 1 & \\
\hline$S_{u s, u s}$ & -0.282 & 0.398 & -0.298 & 1 \\
\hline 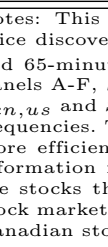 & $\begin{array}{l}\text { le summ } \\
\text { fficiency } \\
\text { requencie } \\
\text {, cn and } \\
\text {,us have } \\
\text { implies } \\
\text { in the O }\end{array}$ & $\begin{array}{l}\text { es the pa } \\
\text { sures, } S_{i} \\
\text { cross the } \\
c n \text { have } \\
\text { second his } \\
\text { the stock } \\
\text { lian stock } \\
\text { o the U. } \\
\text { rmation }\end{array}$ & $\begin{array}{l}\text { wise corre } \\
\text { 's, at } 1-, 5 \\
\text { frequencie } \\
\text { e highest c } \\
\text { est correla } \\
\text { that trans } \\
\text { market wil } \\
\text { market }\end{array}$ & $\begin{array}{l}\text { ations amol } \\
10,-15-, 30 \\
\text { as shown } \\
\text { rrelations ar } \\
\text { ons at vario } \\
\text { it informatic } \\
\text { also transm } \\
\text { ets. Similarl } \\
\text { y in the U. }\end{array}$ \\
\hline
\end{tabular}


Table 9 Basic Statistics for Continuous Variables in Panel Analysis

\begin{tabular}{|c|c|c|c|}
\hline & & Canada & U.S. \\
\hline \multirow{4}{*}{ Market-cap (million U.S. \$) } & Average & \multicolumn{2}{|c|}{$\$ 11,632$} \\
\hline & Std. Dev. & \multicolumn{2}{|c|}{$\$ 21,154$} \\
\hline & 5 th percentile & \multicolumn{2}{|c|}{$\$ 48$} \\
\hline & 95th percentile & \multicolumn{2}{|c|}{$\$ 51,094$} \\
\hline \multirow{4}{*}{ Volume (in sample period) } & Average & $344,550,406$ & $581,653,482$ \\
\hline & Std. Dev. & $435,826,460$ & $1,014,821,171$ \\
\hline & 5 th percentile & $5,119,670$ & $17,166,582$ \\
\hline & 95th percentile & $1,360,727,260$ & $2,681,929,165$ \\
\hline \multirow{4}{*}{ Volume (per minute) } & Average & 4,488 & 5,283 \\
\hline & Std. Dev. & 4,705 & 7,779 \\
\hline & 5 th percentile & 591 & 595 \\
\hline & 95th percentile & 15,180 & 20,869 \\
\hline \multirow{4}{*}{ MediumTrade } & Average & 18,278 & 10,731 \\
\hline & Std. Dev. & 22,667 & 14,462 \\
\hline & 5 th percentile & 353 & 890 \\
\hline & 95th percentile & 61,397 & 42,051 \\
\hline
\end{tabular}

Notes: All stocks are corss-listed in the Canadian and U.S. stock markets. Marketcap of a stock does not vary across the markets. Average market-cap is the average capitalization of the 115 stocks in million U.S. dollars. Volume (in sample period) is the average number of shares traded in the sample period for the 115 stocks (in either the Canadian or the U.S. market) from February 14, 2016 to July 28, 2017. Volume (per minute) is the average number of shares traded per minute for the 115 stocks (in either the Canadian or the U.S. market). MediumTrade is the average of the standard deviations of the minute-by-minute trading volumes for the 115 stocks (in either the Canadian or the U.S. market). MediumTrade is a measure for the proportion of medium trades. The smaller the standard deviation, the more medium trades dominate extreme — very large or vary small — trades. 
Table 10 Panel Data Model for Information Efficiency, $\log \left(S_{i, j}\right), i, j=c n, u s$

\begin{tabular}{|c|c|c|c|c|c|c|c|c|}
\hline Variable & Coef. & S. E. & Variable & Coef. & S. E. & Variable & Coef. & S. E. \\
\hline Intercept & -0.302 & 2.014 & & & & & & 0.730 \\
\hline Canada (baseline) & $0.844^{*}$ & 0.468 & Volume $_{c n}$ (baseline) & $0.456^{* *}$ & 0.214 & Financial (basline) & -0.319 & 0.317 \\
\hline$D_{S_{c n u s}} *$ Canada & $-1.431^{* *}$ & 0.585 & $D_{S_{c n, u s}} *$ Volume $_{c n}$ & 0.255 & 0.294 & $D_{S_{c n, u s}} *$ Financial & -0.475 & 0.398 \\
\hline$D_{S_{u s, c n}} *$ Canada & -0.209 & 0.579 & $D_{S_{u s, c n}} *$ Volume $_{c n}$ & $-0.731 * * *$ & 0.278 & $D_{S_{u s, c n}} *$ Financial & 0.468 & 0.641 \\
\hline$D_{S_{u s, u s}} *$ Canada & -1.207 & 0.658 & $D_{S_{u s, u s}} *$ Volume $_{c n}$ & -0.247 & 0.328 & $D_{S_{u s, u s}} *$ Financial & 0.016 & \\
\hline Market-cap (baseline) & $0.116^{*}$ & 0.063 & Volume $_{u s}$ (baseline) & $-0.343^{* * *}$ & 0.112 & Alternext (baseline) & $-0.672^{* *}$ & 0.496 \\
\hline$D_{S_{c n, u s}} *$ Market-cap & $-0.239 * * *$ & 0.088 & $D_{S_{c n, u s}} *$ Volume $_{u s}$ & -0.173 & 0.288 & $D_{S_{c n, u s}} *$ Alternext & 0.338 & 0.667 \\
\hline$D_{S_{u s, c n}} *$ Market-cap & -0.079 & 0.086 & $D_{S_{u s, c n}} *$ Volume $_{u s}$ & $0.782^{* * *}$ & 0.270 & $D_{S_{u s, c n}} *$ Alternext & 0.512 & 0.416 \\
\hline$D_{S_{u s, u s}} *$ Market-cap & $-0.199^{* *}$ & 0.096 & $D_{S_{u s, u s}} *$ Volume $_{u s}$ & 0.294 & 0.325 & «s $*$ Alternext & $1.395^{* * *}$ & 0.494 \\
\hline MediumTrade $_{c n}$ (baseline) & -0.036 & 1.782 & Basic (baseline) & $1.283^{* * *}$ & 0.336 & NASDAQ (baseline) & 0.061 & 0.376 \\
\hline$D_{S_{c n} u} *$ MediumTrade $_{c n}$ & -0.532 & 0.301 & $D_{S_{c n}, u s} *$ Basic & $-2.281^{* * *}$ & 0.448 & $D_{S_{c n, u s}} * \mathrm{NASDAQ}$ & -0.356 & 0.523 \\
\hline$D_{S_{u s, c n}} *$ MediumTrade $_{c n}$ & 0.237 & 0.290 & $D_{S_{u s, c n}} *$ Basic & -0.103 & 0.420 & $D_{S_{u s, c n}} *$ NASDAQ & $0.485^{* * *}$ & 0.162 \\
\hline$D_{S_{u s, u s}} *$ MediumTrade $_{c n}$ & -0.431 & 0.388 & $D_{S_{u s, u s}} *$ Basic & $-2.620^{* * *}$ & 0.492 & $D_{S_{u s, u s}} *$ NASDAQ & -0.362 & 0.595 \\
\hline MediumTrade $_{u s}$ (baseline) & 0.000 & 0.000 & Technology (baseline) & 0.216 & 0.407 & & & \\
\hline$D_{S_{c n}, u s} *$ MediumTrade $_{u s}$ & $-0.806^{* *}$ & 0.329 & $D_{S_{c n u s}} *$ Technology & 0.286 & 0.718 & & & \\
\hline$D_{S_{u s, c n}} *$ MediumTrade $_{u s}$ & -0.344 & 0.312 & $D_{S_{u, c n}} *$ Technology & -0.322 & 0.569 & & & \\
\hline$D_{S_{u s, u s}} *$ MediumTrade $_{u s}$ & 0.636 & 0.397 & $D_{S_{u s, u s}} *$ Technology & -0.035 & 0.737 & & & \\
\hline
\end{tabular}

$\overline{\overline{\text { Notes: }} \text { The fixed effect panel data model for } \log \left(S_{i, j}\right) \text { 's of the } 115 \text { stocks within and across the Canadian and U.S. markets at }}$ 1-, 5-, 10-, 15-, 30-, and 65-minute frequencies. The Arellano-Bond robust standard errors are reported in parentheses.

$* * *-p<0.01, * *-p<0.05, *-p<0.10$

The higher the value of $\log \left(S_{i, j}\right)$ is, the more efficient the information transmits from market $j$ to market $i$. Information transmission efficiency within Canada measured by $\log \left(S_{c n, c n}\right)$ is the baseline case. Other cases with $\log \left(S_{c n, u s}\right), \log \left(S_{u s, c n}\right)$, and $\log \left(S_{u s, u s}\right)$ are captured, respectively, by dummy variables $D_{S_{c n, u s}}, D_{S_{u s, c n}}$, and $D_{S_{u s, u s}}$ in association with other control variables. Price discovery efficiency is higher for Canadian stocks (the baseline case) within Canada but it is lower when innovations transmit from the U.S. to Canada. Price discovery efficiency is higher for large market-cap stocks within Canada but it is lower when innovations transmit from the U.S. to Canada or within the U.S. Medium trades in both Canada and the U.S. do not affect price discovery efficiency within Canada but a higher level of medium trades in the U.S. is associated with higher price discovery efficiency if innovations transmit from the U.S. to Canada. A higher trading volume in Canada increases price discovery efficiency in Canada but it is not the case for the trading volume in the U.S. The stocks in the basic materials sector, but not the technology and financial sectors, have higher price discovery efficiency in Canada. Among all U.S. exchanges, for stocks traded in the NYSE Alternext, price discovery is less efficient within Canada but more efficient within the U.S. For stocks traded in the NASDAQ, price discovery is more efficient if innovations transmit from Canada to the U.S. The above observations support the efficient home market hypothesis and the sector effect hypothesis. 


\section{APPENDIX A Stylized Measure of $D_{i, j, t}$}

In this appendix, we show an stylized example for the price discovery measure $D_{i, j, t}$. For equation A-1, which is reproduced from equation $(6)$ in the text,

$$
\begin{aligned}
\Delta \mathbf{p}_{t}= & \mathbf{\Psi}(L) \mathbf{G}^{-1} \mathbf{G}^{-1} \mathbf{B} \mathbf{v}_{t} \\
= & {\left[\mathbf{D}_{1}(L) \mathbf{D}_{2}(L)\right]\left[\begin{array}{c}
\alpha_{\perp}^{\prime} \\
\beta^{\prime}
\end{array}\right] \mathbf{A}^{-1} \mathbf{B} \mathbf{v}_{t} } \\
= & \mathbf{D}_{1}(L) \alpha_{\perp}^{\prime} \mathbf{A}^{-1} \mathbf{B} \mathbf{v}_{t}+\mathbf{D}_{2}(L) \beta^{\prime} \mathbf{A}^{-1} \mathbf{B} \mathbf{v}_{t} \\
= & \underbrace{\mathbf{D}_{1}(1) \alpha_{\perp}^{\prime} \mathbf{A}_{t}^{-1} \mathbf{B} \mathbf{v}_{t}}_{\text {long-run impact denoted as }}+\underbrace{\left[\mathbf{D}_{1}(L) \alpha_{\perp}^{\prime} \mathbf{A}^{-1} \mathbf{B}-\mathbf{D}_{1}(1) \alpha_{\perp}^{\prime} \mathbf{A}^{-1} \mathbf{B}+\mathbf{D}_{2}(L) \beta^{\prime} \mathbf{A}^{-1} \mathbf{B}\right] \mathbf{v}_{t}}_{\text {pricing error denoted as } \mathbf{\Phi}^{*}(L) \mathbf{v}_{t} \text { with } \boldsymbol{\Phi}^{*}(1) \mathbf{v}_{t}=\mathbf{0}} .
\end{aligned}
$$

We assume the number of lags $L=1$ and then we have

$$
\boldsymbol{\Psi}=\left[\begin{array}{llll}
\psi_{11} & \psi_{12} & \psi_{13} & \psi_{14} \\
\psi_{21} & \psi_{22} & \psi_{23} & \psi_{24} \\
\psi_{31} & \psi_{32} & \psi_{33} & \psi_{34} \\
\psi_{41} & \psi_{42} & \psi_{43} & \psi_{44}
\end{array}\right]
$$

For equation A-1,

$$
\mathbf{G}=\left[\begin{array}{c}
\alpha_{\perp}^{\prime} \\
\beta^{\prime}
\end{array}\right]
$$

where

$$
\beta=[1,-1,0,0]^{\prime} .
$$

We need to define $\alpha_{\perp}^{\prime}$. Recall $\alpha=\left[\alpha_{c n}, \alpha_{u s}, \alpha_{i d c n}, \alpha_{i d u s}\right]^{\prime}$ and, empirically, $\alpha_{c n}<0, \alpha_{u s}>0, \alpha_{i d c n}=0$ and $\alpha_{i d u s}=0$. Therefore, we have

$$
\alpha_{\perp}^{\prime}=\left[\begin{array}{cccc}
-\alpha_{u s} & -\alpha_{c n} & 0 & 0 \\
0 & 0 & 1 & 0 \\
0 & 0 & 0 & 1
\end{array}\right]
$$

such that $\alpha_{\perp}^{\prime} \alpha=0$. Substituting equations $\mathrm{A}-4$ and $\mathrm{A}-5$ into equation $\mathrm{A}-3$ yields

$$
\mathbf{G}=\left[\begin{array}{cccc}
-\alpha_{u s} & -\alpha_{c n} & 0 & 0 \\
0 & 0 & 1 & 0 \\
0 & 0 & 0 & 1 \\
1 & -1 & 0 & 0
\end{array}\right]
$$

We select a stylized $\mathbf{A}$ and $\mathbf{B}$ :

$$
\mathbf{A}=\left[\begin{array}{cccc}
1 & 0 & A_{c n, i d c n} & A_{c n, i d u s} \\
A_{u s, c n} & 1 & A_{u s, i d c n} & A_{u s, i d u s} \\
0 & 0 & 1 & A_{\text {idcn,idus }} \\
0 & 0 & 0 & 1
\end{array}\right]
$$

and

$$
\mathbf{B}=\mathbf{I}_{4}
$$

The i.i.d. structural shocks are given by

$$
\mathbf{v}=\left[\begin{array}{c}
v_{c n} \\
v_{u s} \\
v_{i d c n} \\
v_{i d u s}
\end{array}\right]
$$

Then we obtain

$$
\mathbf{D}_{1}=\left[\begin{array}{lll}
-\psi_{11} /\left(\alpha_{c n}+\alpha_{u s}\right)-\psi_{12} /\left(\alpha_{c n}+\alpha_{u s}\right) & \psi_{13} & \psi_{14} \\
-\psi_{21} /\left(\alpha_{c n}+\alpha_{u s}\right)-\psi_{22} /\left(\alpha_{c n}+\alpha_{u s}\right) & \psi_{23} & \psi_{24} \\
-\psi_{31} /\left(\alpha_{c n}+\alpha_{u s}\right)-\psi_{32} /\left(\alpha_{c n}+\alpha_{u s}\right) & \psi_{33} & \psi_{34} \\
-\psi_{41} /\left(\alpha_{c n}+\alpha_{u s}\right)-\psi_{42} /\left(\alpha_{c n}+\alpha_{u s}\right) & \psi_{43} & \psi_{44}
\end{array}\right]
$$




$$
\mathbf{D}_{2}=\left[\begin{array}{l}
\alpha_{c n} \psi_{11} /\left(\alpha_{c n}+\alpha_{u s}\right)-\left(\alpha_{u s} \psi_{12}\right) /\left(\alpha_{c n}+\alpha_{u s}\right) \\
\alpha_{c n} \psi_{21} /\left(\alpha_{c n}+\alpha_{u s}\right)-\left(\alpha_{u s} \psi_{22}\right) /\left(\alpha_{c n}+\alpha_{u s}\right) \\
\alpha_{c n} \psi_{31} /\left(\alpha_{c n}+\alpha_{u s}\right)-\left(\alpha_{u s} \psi_{32}\right) /\left(\alpha_{c n}+\alpha_{u s}\right) \\
\alpha_{c n} \psi_{41} /\left(\alpha_{c n}+\alpha_{u s}\right)-\left(\alpha_{u s} \psi_{42}\right) /\left(\alpha_{c n}+\alpha_{u s}\right)
\end{array}\right]
$$

The permanent shocks in this stylized example are given by

$$
\mathbf{D}_{1} \alpha_{\perp}^{\prime} \mathbf{A}^{-1} \mathbf{B v}=\left[\text { permanent }_{1}, \text { permanent }_{2}, \text { permanent }_{3}, \text { permanent }_{4}\right]^{\prime} .
$$

Please note that each permanent shock is a weighted average of the i.i.d. structural shocks, $v_{c n}, v_{u s}, v_{i d c n}$, and $v_{i d u s}$. as shown below:

$$
\begin{gathered}
\text { Permanent }_{1}=-a_{c n}\left(-\frac{\psi_{12}}{a_{u s}+a_{c n}}-\frac{\psi_{11}}{a_{u s}+a_{c n}}\right) v_{u s} \\
+\left(-\left(A_{c n, i d c n} A_{i d c n, i d u s}-A_{c n, i d u s}\right) a_{u s}\left(-\frac{\psi_{12}}{a_{u s}+a_{c n}}-\frac{\psi_{11}}{a_{u s}+a_{c n}}\right)\right.
\end{gathered}
$$

$\left.-\left(-A_{u s, i d u s}+A_{i d c n, i d u s} A_{u s, i d c n}-A_{c n, i d c n} A_{i d c n, i d u s} A_{u s, c n}+A_{c n, i d u s} A_{u s, c n}\right) a_{c n}\left(-\frac{\psi_{12}}{a_{u s}+a_{c n}}-\frac{\psi_{11}}{a_{u s}+a_{c n}}\right)-\psi_{13} A_{\text {idcn,idus }}+\psi_{14}\right) v_{i d u s}$

$$
\begin{aligned}
+\left(A_{c n, i d c n} a_{u s}(\right. & \left.\left.-\frac{\psi_{12}}{a_{u s}+a_{c n}}-\frac{\psi_{11}}{a_{u s}+a_{c n}}\right)-\left(A_{c n, i d c n} A_{u s, c n}-A_{u s, i d c n}\right) a_{c n}\left(-\frac{\psi_{12}}{a_{u s}+a_{c n}}-\frac{\psi_{11}}{a_{u s}+a_{c n}}\right)+\psi_{13}\right) v_{i d c n} \\
& +\left(A_{u s, c n} a_{c n}\left(-\frac{\psi_{12}}{a_{u s}+a_{c n}}-\frac{\psi_{11}}{a_{u s}+a_{c n}}\right)-a_{u s}\left(-\frac{\psi_{12}}{a_{u s}+a_{c n}}-\frac{\psi_{11}}{a_{u s}+a_{c n}}\right)\right) v_{c n}
\end{aligned}
$$

$$
\text { Permanent }_{2}=-a_{c n}\left(-\frac{\psi_{22}}{a_{u s}+a_{c n}}-\frac{\psi_{21}}{a_{u s}+a_{c n}}\right) v_{u s}
$$

$$
+\left(-\left(A_{c n, i d c n} A_{i d c n, i d u s}-A_{c n, i d u s}\right) a_{u s}\left(-\frac{\psi_{22}}{a_{u s}+a_{c n}}-\frac{\psi_{21}}{a_{u s}+a_{c n}}\right)\right.
$$

$\left.-\left(-A_{u s, i d u s}+A_{i d c n, i d u s} A_{u s, i d c n}-A_{c n, i d c n} A_{i d c n, i d u s} A_{u s, c n}+A_{c n, i d u s} A_{u s, c n}\right) a_{c n}\left(-\frac{\psi_{22}}{a_{u s}+a_{c n}}-\frac{\psi_{21}}{a_{u s}+a_{c n}}\right)-\psi_{23} A_{i d c n, i d u s}+\psi_{24}\right) v_{i d u s}$

$$
\begin{gathered}
+\left(A_{c n, i d c n} a_{u s}\left(-\frac{\psi_{22}}{a_{u s}+a_{c n}}-\frac{\psi_{21}}{a_{u s}+a_{c n}}\right)-\left(A_{c n, i d c n} A_{u s, c n}-A_{u s, i d c n}\right) a_{c n}\left(-\frac{\psi_{22}}{a_{u s}+a_{c n}}-\frac{\psi_{21}}{a_{u s}+a_{c n}}\right)+\psi_{23}\right) v_{i d c n} \\
+\left(A_{u s, c n} a_{c n}\left(-\frac{\psi_{22}}{a_{u s}+a_{c n}}-\frac{\psi_{21}}{a_{u s}+a_{c n}}\right)-a_{u s}\left(-\frac{\psi_{22}}{a_{u s}+a_{c n}}-\frac{\psi_{21}}{a_{u s}+a_{c n}}\right)\right) v_{c n}
\end{gathered}
$$

$$
\begin{gathered}
\text { Permanent }_{3}=-a_{c n}\left(-\frac{\psi_{32}}{a_{u s}+a_{c n}}-\frac{\psi_{31}}{a_{u s}+a_{c n}}\right) v_{u s} \\
+\left(-\left(A_{c n, i d c n} A_{i d c n, i d u s}-A_{c n, i d u s}\right) a_{u s}\left(-\frac{\psi_{32}}{a_{u s}+a_{c n}}-\frac{\psi_{31}}{a_{u s}+a_{c n}}\right)\right.
\end{gathered}
$$

$\left.-\left(-A_{u s, i d u s}+A_{i d c n, i d u s} A_{u s, i d c n}-A_{c n, i d c n} A_{i d c n, i d u s} A_{u s, c n}+A_{c n, i d u s} A_{u s, c n}\right) a_{c n}\left(-\frac{\psi_{32}}{a_{u s}+a_{c n}}-\frac{\psi_{31}}{a_{u s}+a_{c n}}\right)-\psi_{33} A_{i d c n, i d u s}+\psi_{34}\right) v_{i d u s}$

$$
\begin{aligned}
+\left(A_{c n, i d c n} a_{u s}(\right. & \left.\left.-\frac{\psi_{32}}{a_{u s}+a_{c n}}-\frac{\psi_{31}}{a_{u s}+a_{c n}}\right)-\left(A_{c n, i d c n} A_{u s, c n}-A_{u s, i d c n}\right) a_{c n}\left(-\frac{\psi_{32}}{a_{u s}+a_{c n}}-\frac{\psi_{31}}{a_{u s}+a_{c n}}\right)+\psi_{33}\right) v_{i d c n} \\
& +\left(A_{u s, c n} a_{c n}\left(-\frac{\psi_{32}}{a_{u s}+a_{c n}}-\frac{\psi_{31}}{a_{u s}+a_{c n}}\right)-a_{u s}\left(-\frac{\psi_{32}}{a_{u s}+a_{c n}}-\frac{\psi_{31}}{a_{u s}+a_{c n}}\right)\right) v_{c n}
\end{aligned}
$$




$$
\begin{gathered}
\text { Permanent }_{4}=-a_{c n}\left(-\frac{\psi_{42}}{a_{u s}+a_{c n}}-\frac{\psi_{41}}{a_{u s}+a_{c n}}\right) v_{u s} \\
+\left(-\left(A_{c n, i d c n} A_{i d c n, i d u s}-A_{c n, i d u s}\right) a_{u s}\left(-\frac{\psi_{42}}{a_{u s}+a_{c n}}-\frac{\psi_{41}}{a_{u s}+a_{c n}}\right)\right. \\
\left.-\left(-A_{u s, i d u s}+A_{i d c n, i d u s} A_{u s, i d c n}-A_{c n, i d c n} A_{i d c n, i d u s} A_{u s, c n}+A_{c n, i d u s} A_{u s, c n}\right) a_{c n}\left(-\frac{\psi_{42}}{a_{u s}+a_{c n}}-\frac{\psi_{41}}{a_{u s}+a_{c n}}\right)-\psi_{43} A_{i d c n, i d u s}+\psi_{44}\right) v_{i d u s} \\
+\left(A_{c n, i d c n} a_{u s}\left(-\frac{\psi_{42}}{a_{u s}+a_{c n}}-\frac{\psi_{41}}{a_{u s}+a_{c n}}\right)-\left(A_{c n, i d c n} A_{u s, c n}-A_{u s, i d c n}\right) a_{c n}\left(-\frac{\psi_{42}}{a_{u s}+a_{c n}}-\frac{\psi_{41}}{a_{u s}+a_{c n}}\right)+\psi_{43}\right) v_{i d c n} \\
+\left(A_{u s, c n} a_{c n}\left(-\frac{\psi_{42}}{a_{u s}+a_{c n}}-\frac{\psi_{41}}{a_{u s}+a_{c n}}\right)-a_{u s}\left(-\frac{\psi_{42}}{a_{u s}+a_{c n}}-\frac{\psi_{41}}{a_{u s}+a_{c n}}\right)\right) v_{c n}
\end{gathered}
$$

Under the condition $L=1$, the transitory shocks are given by

$$
\begin{aligned}
\mathbf{D}_{1} \beta^{\prime} \mathbf{A}^{-1} \mathbf{B v} & =\left[\text { transitor } y_{1}, \text { transitor } y_{2},{\text { transitor } \left.y_{3}, \text { transitor } y_{4}\right]^{\prime}}=[0,0,0,0]^{\prime} .\right.
\end{aligned}
$$

Based on the permanent-transitory decomposition given in equation (A-1) as in equation (6) in the text, we propose a new dynamic price discovery measure for innovations transmitting from market $j$ to market $i$ in period $t$, for $t=0,1,2, \ldots$,

$$
D_{i, j, t}=\frac{\boldsymbol{\Phi}_{i, j}+\boldsymbol{\Phi}^{*}(t)_{i, j}}{\boldsymbol{\Phi}_{i, j}}
$$

where $\boldsymbol{\Phi}_{i, j}\left(\boldsymbol{\Phi}^{*}(t)_{i, j}\right)$ is the $i, j$-th element of $\boldsymbol{\Phi}\left(\boldsymbol{\Phi}^{*}(t)\right)$. This measure is the ratio of the sum of the total impact of innovations, including both the long-term impact and pricing errors, from market $j$ to market $i$ on the price to the long-term impact of innovations from market $j$ to market $i$ on the price in period $t$.

In this stylized example, all transitory shocks will not have any impact while all permanent shocks will have immediate impacts on $\boldsymbol{\Delta} \mathbf{p}_{t}$, where $\mathbf{p}_{t}=\left[p_{c n}, p_{u s}, p_{i d c a n}, p_{i d u s}\right]^{\prime}$. If we are interested in price discovery in $p_{c n}$ caused by $v_{u s}$ at time 0 , we could use the price discovery measure, $D_{i, j, t}$, as follows:

$$
\begin{aligned}
D_{c n, u s, 0} & =\frac{\boldsymbol{\Phi}_{c n, u s}+\boldsymbol{\Phi}^{*}(0)_{c n, u s}}{\boldsymbol{\Phi}_{c n, u s}} \\
& =\frac{-a_{c n}\left(-\frac{\psi_{12}}{a_{u s}+a_{c n}}-\frac{\psi_{11}}{a_{u s}+a_{c n}}\right)+0}{-a_{c n}\left(-\frac{\psi_{12}}{a_{u s}+a_{c n}}-\frac{\psi_{11}}{a_{u s}+a_{c n}}\right)} \\
& =1 .
\end{aligned}
$$

If we are interested in price discovery in $p_{u s}$ caused by $v_{c n}$ at time 0 , we could use the price discovery measure, $D_{i, j, t}$, as follows:

$$
\begin{aligned}
D_{u s, c n, 0} & =\frac{\boldsymbol{\Phi}_{u s, c n}+\boldsymbol{\Phi}^{*}(0)_{u s, c n}}{\boldsymbol{\Phi}_{u s, c n}} \\
& =\frac{\left(A_{u s, c n} a_{c n}\left(-\frac{\psi_{22}}{a_{u s}+a_{c n}}-\frac{\psi_{21}}{a_{u s}+a_{c n}}\right)-a_{u s}\left(-\frac{\psi_{22}}{a_{u s}+a_{c n}}-\frac{\psi_{21}}{a_{u s}+a_{c n}}\right)\right)+0}{\left(A_{u s, c n} a_{c n}\left(-\frac{\psi_{22}}{a_{u s}+a_{c n}}-\frac{\psi_{21}}{a_{u s}+a_{c n}}\right)-a_{u s}\left(-\frac{\psi_{22}}{a_{u s}+a_{c n}}-\frac{\psi_{21}}{a_{u s}+a_{c n}}\right)\right)} \\
& =1 .
\end{aligned}
$$

When the market-specific shocks are the same or $v_{u s}=v_{c n}$, we can compare the long-run role of price discovery by comparing $\boldsymbol{\Phi}_{c n, u s}$ with $\boldsymbol{\Phi}_{u s, c n}$ in the ratio form:

$$
\frac{\boldsymbol{\Phi}_{c n, u s}}{\boldsymbol{\Phi}_{u s, c n}}=\frac{-a_{c n}\left(-\frac{\psi_{12}}{a_{u s}+a_{c n}}-\frac{\psi_{11}}{a_{u s}+a_{c n}}\right)}{\left(A_{u s, c n} a_{c n}\left(-\frac{\psi_{22}}{a_{u s}+a_{c n}}-\frac{\psi_{21}}{a_{u s}+a_{c n}}\right)-a_{u s}\left(-\frac{\psi_{22}}{a_{u s}+a_{c n}}-\frac{\psi_{21}}{a_{u s}+a_{c n}}\right)\right)} .
$$

As the above expression shows, the information channel $\left(A_{u s, c n}\right)$, the error correction coefficients $\left(a_{c n}\right.$ and $\left.a_{u s}\right)$, and moving average coefficients $\left(\psi_{12}, \psi_{12}, \psi_{21}, \psi_{22}\right)$ all play a role. When $L=1,2, \ldots$, the transitory shocks will have some noticeable impact on prices but will vanish over time. As this stylized example shows, the dynamic price discovery measure, $D_{i, j, t}$, will recover more information than $\frac{\left|\alpha_{c n}\right|}{\left|\alpha_{u s}\right|}$ does. The dynamic price discovery measure is affected by the generic moving average representation of reduced form residuals over $L$ lags $(\Psi(L))$, the decomposition of error correction terms and integration terms $(\mathbf{G})$, and the information channels in the markets (A and $\mathbf{B})$. 